Direct numerical simulations of two-phase laminar jet flows with different cross-section injection geometries

H. Abdel-Hameed, and J. Bellan

Citation: Physics of Fluids 14, 3655 (2002); doi: 10.1063/1.1504712

View online: http://dx.doi.org/10.1063/1.1504712

View Table of Contents: http://aip.scitation.org/toc/phf/14/10

Published by the American Institute of Physics

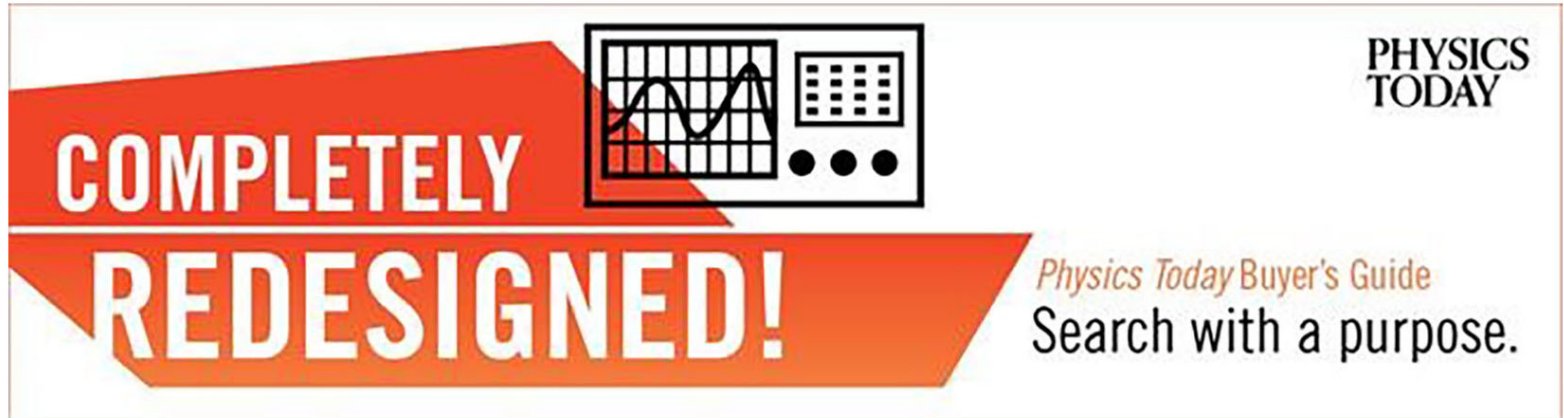




\title{
Direct numerical simulations of two-phase laminar jet flows with different cross-section injection geometries
}

\author{
H. Abdel-Hameed ${ }^{\text {a) }}$ \\ California Institute of Technology, Pasadena, California 91125 \\ J. Bellan ${ }^{\text {b) }}$ \\ Jet Propulsion Laboratory, California Institute of Technology, Pasadena, California 91109
}

(Received 8 November 2001; accepted 15 June 2002; published 5 September 2002)

\begin{abstract}
Direct numerical simulations are performed of spatial, three-dimensional, laminar jets of different inlet geometric configurations for the purpose of quantifying the characteristics of the flows; both single-phase (SP) and two-phase (TP) free jets are considered. The TP jets consist of gas laden with liquid drops randomly injected at the inlet. Drop evaporation ensues both due to the gaseous flow being initially unvitiated by the vapor species corresponding to the liquid drops, and to drop heating as the initial drop temperature is lower than that of the carrier gas. The conservation equations for the TP flow include complete couplings of mass, momentum, and energy based on thermodynamically self-consistent specification of the vapor enthalpy, internal energy, and latent heat of vaporization. Inlet geometries investigated are circular, elliptic, rectangular, square, and triangular. The results focus both on the different spreading achieved according to the inlet geometry, as well as on the considerable change in the flow field due to the presence of the drops. The most important consequence of the drop interaction with the flow is the production of streamwise vorticity that alters entrainment and species mixing according to the inlet geometry. Similar to their SP equivalent, TP jets are shown to reach steady-state entrainment; examination of the flows at this time station shows that the potential cores of TP jets are shorter by an order of magnitude than their SP counterpart. Moreover, whereas the TP circular jet exhibits a symmetric entrainment pattern well past the streamwise location of the potential core, noncircular jets display at the same location strong departures from symmetry. Furthermore, the SP-jet phenomenon of axis switching is no longer present in TP jets. The distributions of drop-number density, liquid mass, and evaporated species are compared for different inlet cross sections and recommendations are made regarding the optimal choice for different applications. (C) 2002 American Institute of Physics.
\end{abstract}

[DOI: $10.1063 / 1.1504712$ ]

\section{INTRODUCTION}

Mixing of particle-laden flows with a flowing gas is a subject of considerable interest for the prediction of both natural phenomena and industrial processes. An example fitting in the first category is the atmospheric dispersion from industrial stacks, whereas an example of the latter is spray dispersion from either household or medical spray cans, or from sprays used in power producing devices. Appropriate mixing affects dispersion patterns and can impact pollutant distribution or the economic and efficient dispersal of liquid products. For hydrocarbon sprays used in power producing devices, spray dispersion influences pollutant formation, particularly soot. Sangiovanni and Liscinsky ${ }^{1}$ have shown that the distance between drops plays a major role in the quantity of soot produced during combustion.

It has been known for some time that single-phase (SP) flow jets with noncircular inlets are characterized by their inherent ability to entrain more fluid than those having cir-

\footnotetext{
a)Permanent address: Zagazig University, Zagazig, Egypt.

${ }^{b)}$ Author to whom correspondence should be addressed: josette.bellan@jpl.nasa.gov
}

cular inlets. The experimental studies of Gutmark et al., ${ }^{2}$ Schadow et al., ${ }^{3}$ Gutmark and Ho, ${ }^{4}$ Ho and Gutmark, ${ }^{5}$ Gutmark et al., ${ }^{6}$ Hussain and Husain, ${ }^{7}$ Husain and Hussain, ${ }^{8}$ Gollahalli et al., ${ }^{9}$ Grinstein et al., ${ }^{10}$ and Zaman ${ }^{11,12}$ have established numerous features of the mixing enhancement of noncircular jets. These jets were either elliptic or issued from corner-containing geometries. Their superior mixing ability was attributed to the accompanying secondary flows that result either from the curvature variation in the azimuthal direction, or from the instabilities produced by the corners through the asymmetric distribution of pressure and mean flow field. In both cases, the coherence of the jet structures is destroyed (e.g., Schadow et al. ${ }^{3}$ ), resulting in larger mixing. For elliptic jets, azimuthal curvature variation of vortical structures produces nonuniform self-induction and threedimensional (3D) structures, resulting in spreading rates becoming larger in the minor axis plane compared to the major axis, thus producing axis switching. Axis switching occurs when the spreading in one direction (e.g., the minor axis plane for elliptic jets) is initially much higher than that in the perpendicular direction (major axis plane for elliptic jets), causing the axis of the cross section to switch ${ }^{7}$ (for elliptic 
jets, the minor axis becomes the major axis, so that the ellipse appears rotated by $90^{\circ}$ ). Depending on the specific corner-containing geometry, the axis switching phenomenon might not necessarily happen (equilateral triangle inlets display axis switching, but square inlets do not), however, the corners promote the formation of fine-scale turbulence, which enhances mixing. These experimental results were confirmed by a large number of numerical predictions. Examples of such computational studies are those of Koshigoe et al. ${ }^{13}$ for triangular jets, Grinstein and DeVore, ${ }^{14}$ Grinstein and Kailasanath, ${ }^{15}$ and Grinstein et al. ${ }^{10}$ for square jets, Tam and Thies ${ }^{16}$ and Grinstein ${ }^{17}$ for rectangular jets, and Miller et al. ${ }^{18}$ for several jet geometries. All of these studies were devoted to SP flows and were either based on stability analysis $^{13,16}$ leading to a turbulent state, or on an inviscid approach where the turbulent model was effectively provided by the numerical dissipation. ${ }^{14,15,18}$ Husain and Hussain ${ }^{19}$ used direct numerical simulation (DNS) in their theoretical study of elliptic jets and found that unlike in circular jets, forcing was not necessary for elliptic jets to induce formation of azimuthally fixed streamwise vortices. Rembold et al. ${ }^{20}$ also used DNS but for rectangular jets, and like Husain and Hussain ${ }^{19}$ found a rapid transition to small-scale turbulence. Jiang and Luo ${ }^{21}$ devoted most of their spatial DNS study of rectangular buoyant gaseous free jets to studying combustion and buoyancy effects on the tendency to transition, although some nonreacting simulations were discussed for comparison. The recent review of Gutmark and Grinstein ${ }^{22}$ summarizes both experimental and numerical studies in the context of noncircular jets.

In contrast to all previous studies, the present investigation is devoted to two-phase (TP) flows, and examines only the laminar regime. The intention here is to investigate whether the superior mixing characteristics of noncircular SP jets translate to the TP situation. If this is found to occur herein and is further confirmed for turbulent situations, a change in injection geometry would constitute a strategy for enhancing spray dispersion and furthermore promote mixing of the evaporated species with the ambient gas.

To date, the modeling of TP gas-liquid turbulent flows has proven to be difficult due to the complexity associated with the interaction between the carrier gas and the drops. Recently, Miller and Bellan ${ }^{23,24}$ and Okong'o and Bellan ${ }^{25}$ have addressed the configuration of a 3D mixing layer initially laden with drops in the lower stream. The focus of that previous work was first on the understanding of the interaction between drops and gas in a pre-transitional setting, and then on the creation of transitional states that served as databases from which small scale turbulent models were extracted. The present work adopts a similar strategy for 3D jets, and therefore the focus is here on the pre-transitional features of drop laden jets having different inlet geometries.

The paper is organized as follows: In Sec. II we briefly recall some aspects of the formulation of Miller and Bellan $^{23,24}$ and highlight the differences in the modeling of the heat and mass fluxes between the present formulation and the aforementioned work. Furthermore, we explain the departures in the treatment of boundary conditions between the confined mixing layer investigated in the previous studies and the present investigation of a free jet. The numerical procedure is briefly addressed in Sec. III. The initial conditions are documented in Sec. IV. Section V is devoted to the discussion of the results, exploring the differences between SP jets and TP jets, and those between circular and noncircular jets. Finally, in the last section we state our conclusions and make recommendations for further investigations.

\section{MODEL}

The mathematical description of TP flows has followed two distinct methods. The first, mainly reserved for dilute flows (i.e., small condensed-phase volume fraction), is the Eulerian-Lagrangian method whereby the gas is described in the frame of the observer (i.e., Eulerian frame) and the condensed particles are followed on their trajectories (i.e., Lagrangian frame). In the other method, both gas and condensed phase are considered as continuum flows, and they are both followed in the Eulerian frame. The present approach adopts the former technique, emulating the study of Miller and Bellan ${ }^{23,24}$ in the context of mixing layers. The conservation equations are solved in the framework of DNS originally introduced for SP flows, meaning that all length and time scales of the flow are resolved without resorting to either time averaged or subgrid turbulence models. In this respect, even the present SP flow simulations are different from those of Grinstein and DeVore, ${ }^{14}$ Grinstein and Kailasanath, ${ }^{15}$ Miller et al. ${ }^{18}$ and Grinstein, ${ }^{17}$ which all contained numerical dissipation that acted akin to subgrid fluctuations. Therefore, the present SP simulations are closer in spirit to the (incompressible, periodic-box spectral) DNS of Husain and Hussain, ${ }^{19}$ which were however for a much larger initial Reynolds number, $\operatorname{Re}_{0}=1500$, and of Rembold et $a .^{20}$ at a Reynolds number of 2000; their investigations were performed at larger Reynolds numbers than the present simulations, as no computational resources were needed to follow the drops.

Noteworthy, in the realm of TP flows, the term DNS has been coined by Squires and Eaton ${ }^{26,27}$ to mean that all length scales of the flow are resolved, but that the condensed-phase particles are treated as point sources. This terminology reflects the current impossibility (due to computer memory and computational time constraints) of solving the exact equations inside the drops and in their immediate vicinity when tracking a very large number of drops [e.g., $\left.\sim O\left(10^{5}\right)-O\left(10^{6}\right)\right]$, even if the calculations are performed in a small domain and for a relatively small $(\sim 400) \mathrm{Re}_{0}$. This situation led to assuming the drop size to be much smaller than the Kolmogorov scale, $\eta_{K}$, and treating them as point forces and sources. Liljegren ${ }^{28}$ noted that for solid particles in homogeneous flow (mean fluid and particle velocities are constant and linear, and the density of the condensed phase is spatially uniform and does not vary with time) this point force/source assumption leads to losing the effect of particles on the fluctuating kinetic energy of the carrier flow to leading order; however, this effect might be more pronounced for solid particles in truly turbulent flows, which are not the present focus. 
Additional to the point-source assumption, other restrictions are here imposed to make the problem computationally tractable: (i) the drops are spherical, (ii) the ensemble of drops has negligible volume fraction (see the a posteriori verification in Sec. VD), and (iii) there are no interactions among drops (i.e., there is no drop collision, breakup, or coalescence). The gas-phase equations are completely coupled to the drop equations in that mass, momentum and energy are exchanged between the two phases. Just as in Miller and Bellan, ${ }^{23,24}$ we describe the single drop evolution using the validated model of Miller et al. ${ }^{29}$ In this model, the momentum coupling between drops and flow occurs through the drag force (i.e., Basset history, added mass, lift, Magnus, and other forces are neglected), and the internal droplet temperature is uniform, with heat transfer to the drop occurring through convection and conduction.

\section{A. Gas-phase conservation equations}

The compressible form of the governing equations for the gas phase, including mass, momentum, and energy coupling are

$$
\begin{aligned}
& \frac{\partial}{\partial t}\left(\rho_{G}\right)+\frac{\partial}{\partial x_{j}}\left[\rho_{G} u_{j}\right]=S_{I}, \\
& \frac{\partial}{\partial t}\left(\rho_{G} u_{i}\right)+\frac{\partial}{\partial x_{j}}\left[\rho_{G} u_{i} u_{j}+p_{G} \delta_{i j}-\tau_{i j}\right]=S_{I I, i}, \\
& \frac{\partial}{\partial t}\left(\rho_{G} E_{G}\right)+\frac{\partial}{\partial x_{j}}\left[\left(\rho_{G} E_{G}+p_{G}\right) u_{j}-q_{j}-u_{i} \tau_{i j}\right]=S_{I I I}, \\
& \frac{\partial}{\partial t}\left(\rho_{G} Y_{V}\right)+\frac{\partial}{\partial x_{j}}\left[\rho_{G} Y_{V} u_{j}-j_{V j}\right]=S_{I}, \\
& p_{G}=\rho_{G} R_{u}\left[\frac{Y_{V}}{W_{V}}+\frac{\left(1-Y_{V}\right)}{W_{C}}\right] T_{G},
\end{aligned}
$$

where $q_{j}$ is the heat flux, $j_{V j}$ is the species mass flux, $\rho_{G}$ is the gas-phase density, $u_{i}$ is the gas-phase velocity, $p_{G}$ is the thermodynamic pressure, $T_{G}$ is the gas temperature, $\tau_{i j}$ is the viscous stress tensor (with viscosity $\mu_{G}$ ), $E_{G}=e_{G}+\frac{1}{2} u_{i} u_{i}$ is the total gas energy (internal $e_{G}$ and kinetic $\frac{1}{2} u_{i} u_{i}$ ), and $Y_{V}$ is the mass fraction of the evaporated liquid species. Also, $W$ denotes the molecular weight, $\delta_{i j}$ is the Kronecker delta function, and $R_{u}$ is the universal gas constant. The right-hand side terms $S_{I}, S_{I I, i}$, and $S_{I I I}$ are the coupling terms between the gas and the condensed phase whose form is given below. Subscripts $C$ and $V$ refer to the carrier gas and the vapor emitted by the drops, respectively.

\section{B. Individual droplet conservation equations}

The Lagrangian equations describing the transient displacement $\left(X_{i}\right)$, velocity $\left(v_{i}\right)$, temperature $\left(T_{d}\right)$, and mass $\left(m_{d}\right)$ of a single droplet are those derived by Miller and Bellan: ${ }^{23}$

$$
\frac{d X_{i}}{d t}=v_{i},
$$

$$
\begin{aligned}
& \frac{d v_{i}}{d t}=\frac{F_{i}}{m_{d}}=\frac{f_{1}}{\tau_{d}}\left(u_{i}-v_{i}\right), \\
& \begin{aligned}
\frac{d T_{d}}{d t}=\frac{Q_{d}+\dot{m}_{d} L_{V}}{m_{d} C_{L}}= & \frac{N u}{3 \operatorname{Pr}_{G}}\left(\frac{C_{p, G}}{C_{L}}\right)\left(\frac{f_{2}}{\tau_{d}}\right)\left(T_{G}-T_{d}\right) \\
& +\left(\frac{\dot{m}_{d}}{m_{d}}\right) \frac{L_{V}}{C_{L}},
\end{aligned} \\
& \dot{m}_{d} \equiv \frac{d m_{d}}{d t}=-\frac{S h}{3 S c_{G}}\left(\frac{m_{d}}{\tau_{d}}\right) \ln \left[1+B_{M}\right],
\end{aligned}
$$

where the subscript $d$ denotes individual drop conditions, $\rho_{L}$ is the liquid density, the droplet time constant for Stokes flow is $\tau_{d}=\rho_{L} d^{2} /\left(18 \mu_{G}\right)$ with $d$ being the droplet diameter, $Q_{d}$ is the heat transfer rate to the drop which is driven by the local difference in temperature between drop and gas, $C_{L}$ is the heat capacity of the liquid, whereas $L_{V}$ is the latent heat of evaporation. The gas mixture heat capacity is calculated as $C_{p, G}=\left(1-Y_{V}\right) C_{p, C}+Y_{V} C_{p, V}$, where $C_{p, C}$ and $C_{p, V}$ are the heat capacities of the carrier gas and vapor, respectively. The gas-phase Prandtl and Schmidt numbers are $\operatorname{Pr}_{G}$ $=\mu_{G} C_{p, G} / \lambda_{G}$ and $S c_{G}=\mu_{G} /\left(\rho_{G} \Gamma_{V}\right)$ where $\Gamma_{G}$ and $\lambda_{G}$ are the Fickian diffusion coefficient and the constant gas-phase thermal conductivity, respectively. The drag force is driven by the local slip velocity vector $u_{s l, i}=u_{i}-v_{i}$ and the Stokes drag in Eq. (7) is empirically corrected for finite droplet Reynolds numbers by the correlation

$$
\begin{aligned}
& f_{1}=\frac{1+0.0545 \mathrm{Re}_{s l}+0.1 \mathrm{Re}_{s l}^{1 / 2}\left(1-0.03 \mathrm{Re}_{s l}\right)}{1+a\left|\mathrm{Re}_{b}\right|^{b}}, \\
& a=0.09+0.07 \exp \left(-0.4 \mathrm{Re}_{s l}\right), \\
& b=0.4+0.77 \exp \left(-0.04 \mathrm{Re}_{s l}\right),
\end{aligned}
$$

where $U_{s l}=\left|u_{i}-v_{i}\right|$ is the slip velocity magnitude, and the blowing velocity, $U_{b}$, is related to the mass flux evaporated from the drop through $\dot{m}_{d}=-\pi \rho_{G} d^{2} U_{b}$. The two characteristic velocities, $U_{s l}$ and $U_{b}$, are used to define two drop Reynolds numbers, $\operatorname{Re}_{s l}=\rho_{G} U_{s l} d / \mu_{G}$ and $\operatorname{Re}_{b}=\rho_{G} U_{b} d l \mu_{G}$. The Nusselt $(\mathrm{Nu})$ and Sherwood $(\mathrm{Sh})$ numbers are empirically modified for convective corrections to heat and mass transfer that are based on the Ranz-Marshall correlations (Ranz and Marshall ${ }^{30,31}$ )

$$
N u=2+0.552 \operatorname{Re}_{s l}^{1 / 2} \operatorname{Pr}_{G}^{1 / 3}, \quad S h=2+0.552 \operatorname{Re}_{s l}^{1 / 2} S c_{G}^{1 / 3} .
$$

The function $f_{2}=\beta /\left(e^{\beta}-1\right)$ is an analytical evaporative heat transfer correction to the solid-sphere Nusselt number, where the nondimensional evaporation parameter $\beta$, given by

$$
\beta=-\left(\frac{3 \operatorname{Pr}_{G} \tau_{d}}{2}\right) \frac{\dot{m}_{d}}{m_{d}}=-\left(\frac{\rho_{L} \operatorname{Pr}_{G}}{8 \mu_{G}}\right) \frac{d\left(d^{2}\right)}{d t},
$$

is constant for drops obeying the " $d^{2}$ law" (see Miller et al. $\left.^{29}\right)$. 


\section{Source terms}

The phase-coupling terms appearing in Eqs. (1)-(4) are

$$
\begin{aligned}
& S_{I}=-\sum_{\alpha}\left\{\frac{w_{\alpha}}{\Delta V_{\alpha}}\left[\dot{m}_{d}\right]_{\alpha}\right\}, \\
& S_{I I, i}=-\sum_{\alpha}\left\{\frac{w_{\alpha}}{\Delta V_{\alpha}}\left[F_{i}+\dot{m}_{d} v_{i}\right]_{\alpha}\right\}, \\
& S_{I I I}=-\sum_{\alpha}\left\{\frac{w_{\alpha}}{\Delta V_{\alpha}}\left[v_{i} F_{i}+Q_{d}+\dot{m}_{d}\left(\frac{v_{i} v_{i}}{2}+h_{V, s}\right)\right]_{\alpha}\right\},
\end{aligned}
$$

where $h_{V, s}$ is the evaporated vapor enthalpy at the droplet surface, and the drag force $F_{i}, Q_{d}$ and $\dot{m}_{d}$ are specified by the modeled droplet conservation equations, Eqs. (7)-(9). The summations are over all droplets residing within a local numerical discretization volume $\Delta V_{\alpha}$ and employ a geometrical weighting factor, $w_{\alpha}$, which is used to proportionally distribute the individual droplet contributions to the eight nearest neighbor surrounding grid points (i.e., corners of the computational volume $\Delta V_{\alpha}$ ) according to the respective distance of the drop from the corners of the computational volume.

\section{Fluxes, internal energy, and latent heat}

According to Hirshfelder et al., ${ }^{32}$ the general form of the molar and heat fluxes, in absence of Soret and Dufour effects (here neglected, owing to the atmospheric pressure conditions), is

$$
\begin{gathered}
J_{V j}=-\rho Y_{V}\left[\frac{\Gamma_{G} \partial Y_{V}}{Y_{V} \partial x_{j}}+Y_{C}\left(Y_{V}+Y_{C} \frac{m_{V}}{m_{C}}\right)\left(\frac{m_{C}}{m_{V}}-1\right) \frac{\Gamma_{G} \partial p_{G}}{p_{G} \partial x_{j}}\right], \\
q_{j}=-\lambda_{G} \frac{\partial T_{G}}{\partial x_{j}}+\left(h_{V}-h_{C}\right) J_{V j},
\end{gathered}
$$

where $j_{V j}=m_{V} J_{V j}$. Here $h_{C}$ and $h_{V}$ are the enthalpies of the pure gases

$$
h_{C}=C_{p, C} T_{G}, \quad h_{V}=C_{p, V} T_{G}+h_{V}^{0},
$$

with the mixture enthalpy defined by

$$
h=e+p / \rho=C_{p} T+h_{V}^{0} Y_{V}=h_{C} Y_{C}+h_{V} Y_{V} .
$$

We note that the present formulation is more general than that of Miller and Bellan ${ }^{23,24}$ in that it includes the pressuregradient term in Eq. (17) and the enthalpy carried by the molar-fluxes term in Eq. (18). Preliminary calculations have shown that the pressure-gradient term in Eq. (17) is small with respect to the species-gradient term, and as a result has been neglected in all simulations presented herein. However, the enthalpy carried by the molar fluxes may rival the thermal-conductivity term under low $T_{G}$ conditions such as those studied herein.

For thermally perfect species and calorically perfect gas (i.e., constant heat capacities at constant pressure)

$$
\begin{aligned}
& h_{V, s}=C_{p, V} T_{d}+h_{V}^{0}, \\
& L_{V}=h_{V}^{0}-\left(C_{L}-C_{p, V}\right) T_{d},
\end{aligned}
$$

$$
e_{G}=\left(1-Y_{V}\right)\left[C_{v, C} T_{G}\right]+Y_{V}\left[C_{v, V} T_{G}+h_{V}^{0}\right] .
$$

The drop temperature is uniform due to the assumption of infinite liquid thermal conductivity, and thus $T_{d, s}=T_{d}$.

Using the above definitions, the phase coupling terms may now be expressed as

$$
\begin{aligned}
& S_{I}=-\sum_{\alpha}\left\{\frac{w_{\alpha}}{\Delta V_{\alpha}}\left[\frac{d}{d t}\left(m_{d}\right)\right]_{\alpha}\right\}, \\
& S_{I I, i}=-\sum_{\alpha}\left\{\frac{w_{\alpha}}{\Delta V_{\alpha}}\left[\frac{d}{d t}\left(m_{d} v_{i}\right)\right]_{\alpha}\right\}, \\
& S_{I I I}=-\sum_{\alpha}\left\{\frac{w_{\alpha}}{\Delta V_{\alpha}}\left[\frac{d}{d t}\left(m_{d} C_{L} T_{d}+\frac{1}{2} m_{d} v_{i} v_{i}\right)\right]_{\alpha}\right\},
\end{aligned}
$$

where the time derivatives express the total rates of change of the individual drop mass, momentum, and total energy.

\section{E. Boundary conditions}

The adopted boundary conditions are based on the Navier-Stokes characteristic boundary conditions (NSCBC) derived by Poinsot and Lele ${ }^{33}$ and Baum et al. ${ }^{34}$ The NSCBC method is justified by the fact that at high Re, the NavierStokes (NS) equations may be considered to be an incompletely elliptic perturbation of the Euler equations, which are hyperbolic. Therefore, the idea is that the inviscid character of the equations dominates, and the viscous aspect is only a perturbation introduced for well-posedness of the mathematical problem once the inviscid conditions are established. According to the Baum et al. ${ }^{34}$ treatment for a mixture of gas and single vapor species, and focusing on the local onedimensional inviscid (LODI) relations for the primitive variables in $x_{1}$ direction, these boundary conditions are

$$
\begin{aligned}
& \frac{\partial \rho}{\partial t}+\frac{1}{c^{2}}\left[\frac{1}{2}\left(\mathcal{L}_{1}+\mathcal{L}_{6}\right)-\mathcal{L}_{2}\right]=0, \\
& \frac{\partial u_{1}}{\partial t}+\frac{\mathcal{L}_{6}-\mathcal{L}_{1}}{2 \rho c}=0, \quad \frac{\partial u_{2}}{\partial t}+\mathcal{L}_{3}=0, \quad \frac{\partial u_{3}}{\partial t}+\mathcal{L}_{4}=0 \\
& \frac{\partial Y_{V}}{\partial t}+\mathcal{L}_{5}=0 \\
& \frac{\partial p_{G}}{\partial t}+\frac{\mathcal{L}_{6}+\mathcal{L}_{1}}{2}=0
\end{aligned}
$$

where the $\mathcal{L}_{i}$ 's are the time variation of the wave amplitude of the primitive variables. Each $\mathcal{L}_{i}$ is thus associated with a wave having the speed $\lambda_{i}$. In the present situation, $\lambda_{1}=u_{1}$ $-c, \lambda_{2}=\lambda_{3}=\lambda_{4}=\lambda_{5}=u_{1}$, and $\lambda_{6}=u_{1}+c$, where $c$ is the sonic speed. From the above relations, the amplitude variation of the characteristic waves is

$$
\begin{gathered}
\mathcal{L}_{1}=\lambda_{1}\left(\frac{\partial p_{G}}{\partial x_{1}}-\rho c \frac{\partial u_{1}}{\partial x_{1}}\right), \\
\mathcal{L}_{2}=\lambda_{2}\left(\frac{\partial p_{G}}{\partial x_{1}}-c^{2} \frac{\partial \rho}{\partial x_{1}}\right),
\end{gathered}
$$




$$
\begin{aligned}
& \mathcal{L}_{3}=\lambda_{3} \frac{\partial u_{2}}{\partial x_{1}}, \quad \mathcal{L}_{4}=\lambda_{4} \frac{\partial u_{3}}{\partial x_{1}}, \quad \mathcal{L}_{5}=\lambda_{5} \frac{\partial Y_{V}}{\partial x_{1}}, \\
& \mathcal{L}_{6}=\lambda_{6}\left(\frac{\partial p_{G}}{\partial x_{1}}+\rho c \frac{\partial u_{1}}{\partial x_{1}}\right) .
\end{aligned}
$$

Since the present simulations are of a 3D free, viscous subsonic jet flow, there is one inflow boundary at $x_{1}=0$, and the other five boundaries conditions are of type outflow.

For physical consistency and well-posedness, the Euler equations must have six boundary conditions at the inflow. Five of these conditions are: the imposed value of the gas density, the vapor mass fraction and the three components of the velocity. The additional "numerical," or "soft," boundary condition is found from the energy equation. From Eqs. (31) - (34), the only amplitude variation of the characteristic waves that exits the domain is $\mathcal{L}_{1}$, which can be calculated from the interior domain using Eq. (31). Using Eqs. (27)(30), and according to the five imposed inflow conditions, leads to

$$
\mathcal{L}_{6}=\mathcal{L}_{1} \text { and } \mathcal{L}_{2}=\mathcal{L}_{1},
$$

with all the other $\mathcal{L}_{i}$ 's being null. These boundary conditions, combined with Eq. (30), enable the finding of a solution for the energy equation at the inflow. Finally, the normal derivative of the normal viscous stress is chosen to be null, expressing the viscous aspect of the NS equations.

At all outflow boundaries and in all directions, we choose subsonic nonreflecting boundary conditions based on the NSCBC derivation above, which was developed for illustrative purposes only in the $x_{1}$ direction. Using NSCBCdeveloped conditions provides the necessary physical support to what would otherwise be ad hoc/uncertain boundary conditions. For the Euler equations, it is sufficient to impose the pressure at infinity, $p_{\infty}$. To ensure well-posedness

$$
\mathcal{L}_{1}=K\left(p-p_{\infty}\right)
$$

where $\mathcal{L}_{1}$ is the only in-going characteristic wave, $K$ is a constant, $K=\sigma\left(1-M_{\max }^{2}\right) c / L, M_{\max }$ is the maximum Mach number in the flow, $L$ is a characteristic size of the domain, and $\sigma$ is a constant. Throughout our computations, $\sigma$ is chosen to be 0.25 . To complete the boundary conditions with the viscous conditions necessary for the NS equations, we choose the normal derivative of the tangential stresses, the normal heat flux, and the normal vapor mass fraction flux to be all null.

At corners, the imposed boundary conditions in a given direction are consistent with the direction of the flow, being either inflow or outflow.

\section{NUMERICS}

The gas-phase equations are discretized using an implicit sixth-order accurate central finite differences (see Kennedy and Carpenter ${ }^{35}$ ) to represent all spatial derivatives in the interior nodes of the domain, and a fifth-order backward/ forward differencing for boundary nodes. The time integration is performed using an explicit fourth-order Runge-Kutta temporal integration for all time derivatives. Each droplet is a physical entity, in contrast to the stochastic representations using computational drops. Because the gas-phase variables are needed at the drop locations to calculate the drop-gas interaction [see Eqs. (6)-(9)], a fourth-order Lagrange interpolation procedure is used to obtain the gas variables which are calculated at nodes, at the position of each drop. The source terms $S_{I}, S_{I I, i}$, and $S_{I I I}$ exhibit spatial "spottiness" which, unless mitigated, would introduce artificial oscillations and destabilize this very low dissipation differencing scheme. To remove this spottiness, the source terms are minimally smoothed with a conservative operator (the volume integrated source terms are unaltered), as explained in detail in Miller and Bellan. ${ }^{23}$ This topic has also been discussed by Tong and Wang ${ }^{36}$ who remarked that the numerical diffusion term necessary to enable the finding of a solution to the otherwise stiff drop number density equation in the Eulerian-Eulerian approach is effectively equivalent to the necessary smoothing of the particle contribution in the Eulerian-Lagrangian approach.

Despite the source smoothing, it is found that due to the high-order central spatial differencing, there is no sufficient numerical diffusion to control the buildup of artificial numerical oscillations in the solution. These errors are primarily due to differencing the nonlinear convective terms, and may become significant at long simulation times. In order to control this problem, a eighth-order explicit filtering is applied to all the primitive variables of the gas field once every five cycles of the Runge-Kutta integration. In this work, we use the complete amplitude suggested by Kennedy and Carpenter, ${ }^{35}$ which is found sufficient to provide dissipation without contaminating the physical domain. The computational grid is uniformly spaced with $\Delta x=\Delta x_{1} \approx \Delta x_{2} \approx \Delta x_{3}$. In all simulations, the grid resolution is $240 \times 180 \times 180$ in the $x_{1}, x_{2}$, and $x_{3}$ directions. Based on previous experience with TP mixing layers (e.g., Miller and Bellan ${ }^{23}$ ), this grid resolution captures very well the laminar simulations of interest in this study.

Specification of the time step for each simulation is based on the consideration of a maximum Courant number of 0.5 . When a drop diameter reaches 0.1 of its initial value, its mass is "frozen," but the drops are still followed on their trajectories. This is in contrast to the mixing layer simulations of Miller and Bellan ${ }^{23,24}$ where the drops reaching this residual size were removed from the computational domain.

The present simulations are conducted on an SGI Origin 2000 parallel supercomputer and Message Passing Interface (MPI) subroutines for interprocessor communications are used. The Eulerian gas phase equations are parallelized using three-dimensional domain decomposition in which all the derivatives, source term smoothing and the filtering operation are performed using the ghost cell methodology. The Lagrangian drop equations are solved by the same individual processors allocated to the Eulerian domain in which the droplets reside; in this manner, the necessary processor communication is greatly reduced. When drops move from one portion of the physical domain to another portion that is calculated by a different processor, these drops and all their properties are accordingly transferred to the different processor. This choice optimizes the CPU time past the initial condition, when some processors are devoid of drops. Past this 


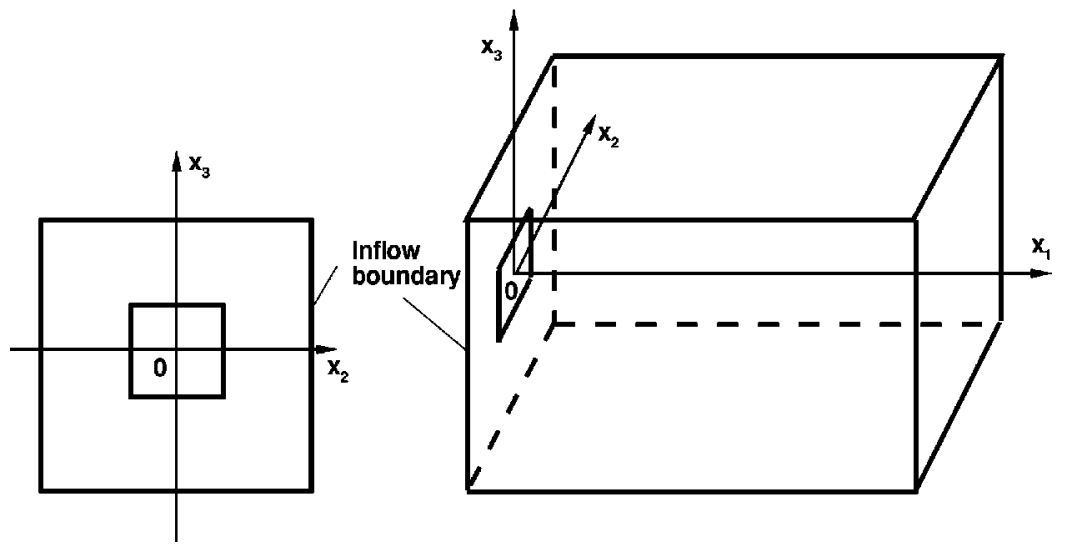

FIG. 1. Geometric configuration, here illustrated for a square cross-section jet.

initial time, the processors contain a similar number of drops, and thus the computational load is well balanced. All simulations are performed using 32 processors with a $8 \times 2 \times 2$ spatial decomposition.

\section{FLOW CONFIGURATION AND INITIAL CONDITIONS}

The 3D geometric configuration is illustrated in Fig. 1 for the special case of a square inlet, and the coordinates $x_{1}$, $x_{2}$, and $x_{3}$ are defined. The computational domain dimensions are $L_{1}=2 L_{2}=2 L_{3}=8 D_{J}=0.16 \mathrm{~m}$. The boundaries of the domain in Fig. 1 are computational, as the jet is unconfined (free jet). The equivalent jet diameter $D_{J}$ (which is the diameter of the circle that has the same area as the noncircular inlet sections) is chosen to be $0.02 \mathrm{~m}$ for all geometric configurations; this means that while the cross-section geometry changes, the area is the same in all simulations. Several noncircular configurations are considered, as listed in Table I. The elliptic configuration is chosen so that the major axis is in the $x_{2}$ direction, symmetrically centered about $x_{3}=0$ and with major and minor axis lengths of $0.028 \mathrm{~m}$ and 0.014 $\mathrm{m}$, respectively. The square configuration is centered at $x_{2}$ $=x_{3}=0$ with sides of $0.0177 \mathrm{~m}$ in length. The rectangle is also centered at $x_{2}=x_{3}=0$ and has its larger side of length $0.025 \mathrm{~m}$ along the $x_{2}$ axis, while its smaller side is $0.0125 \mathrm{~m}$ in length. Finally, the triangle is equilateral, with a peak located at $x_{2}=0 \mathrm{~m}$ and $x_{3}=0.01166 \mathrm{~m}$.

The Mach number is defined as

$$
M=U_{0} / \sqrt{R_{u} T_{G, 0} C_{p, C} /\left(W_{C} C_{v, C}\right)},
$$

where the jet Mach number, $M_{J}=0.35$, is based on $U_{0}$ $=U_{J}$, and the ambient Mach number, $M_{\infty}=0.05$, based on $U_{\infty}$. The velocity $U_{J}=131 \mathrm{~m} / \mathrm{s}$ is the constant inlet gas ve-

TABLE I. Geometries, aspect ratio (AR), and time at which a mass flux steady-state condition was achieved for the single and two-phase flow simulations.

\begin{tabular}{lllc}
\hline \hline Run & \multicolumn{1}{c}{ Geometry } & AR & $t_{s}^{*}=t \Delta U_{0} / D_{j}$ \\
\hline 1SP; 1TP & circular & $1: 1$ & $14.8 ; 13.3$ \\
2SP; 2TP & elliptic & $2: 1$ & $18.2 ; 18.4$ \\
3SP; 3TP & square & $1: 1$ & $14.2 ; 15.4$ \\
4SP; 4TP & rectangular & $2: 1$ & $18.5 ; 21.1$ \\
5SP; 5TP & triangular & $1: 1$ & $17.5 ; 18.5$ \\
\hline \hline
\end{tabular}

locity, being determined from the specified value of $M_{J}$, and $U_{\infty}=18.7 \mathrm{~m} / \mathrm{s}$ is the constant gas velocity in the freestream, being determined from the specified value of $M_{\infty}$. To avoid a spatial discontinuity, the two disparate velocities are connected through a tanh function with a width of $D_{J} / 25$ in the direction of initial discontinuity, $x_{2}$. In Eq. (37), $T_{G, 0}$ is the initial gas temperature and $C_{v, C}$ is the carrier gas heat capacity at constant volume.

The initial jet Reynolds number, $\operatorname{Re}_{0}=\rho_{G, 0} \Delta U_{0} D_{J} / \mu_{G}$, where $\Delta U_{0}=U_{J}-U_{\infty}$, is typically 500, although some single-phase flow simulations were also performed for $\mathrm{Re}_{0}$ $=400$ and 600 to illustrate some features of behavior (the different $\operatorname{Re}_{0}$ values were obtained by varying $\left.\mu_{G}\right)$. The initial flow field is isobaric with $p_{G, 0}=p_{\text {atm }}$, and isothermal with $T_{G, 0}=350 \mathrm{~K}$, and $\rho_{G, 0}=1 \mathrm{~kg} / \mathrm{m}^{3}$. The droplets are inserted into the domain through the jet orifice randomly distributed, and with a velocity of $0.75 U_{J}$, leading to a non-null initial droplet slip velocity. The drop size is uniform (portraying a monodisperse spray) with initial diameter of $50 \mu \mathrm{m}$ and initial temperature $T_{d, 0}=325 \mathrm{~K}$. The injected liquid flux is $\dot{m}_{L}=0.012 \mathrm{~kg} / \mathrm{s}$, leading to a mass loading $M L \equiv \dot{m}_{L} / \dot{m}_{C}$ $=0.29$.

This study is performed with air as the carrier gas, and decane as the liquid. Initially, the freestream mass fraction of the evaporated species is null, and the equivalent value in the jet is 0.03; a tanh function of the same width as that of the initial velocity profile is used to avoid a spatial discontinuity in the mass fraction. All heat capacities, the gas Prandtl number and vapor reference enthalpy are evaluated at $350 \mathrm{~K}$. The Lewis number $\left(L e_{G}\right)$ is assumed to be unity (i.e., $S c_{G}$ $=\operatorname{Pr}_{G}$ ). The gas phase viscosity is determined through the specification of $\operatorname{Re}_{0}$. The constant property values used in the simulations are provided in Table II.

The specification of all initial conditions permits the calculation of the initial Stokes number, which measures the ratio of the drop-to-flow characteristic times. By definition, $S t=\tau_{d} \Delta U_{0} /\left(D_{J} / 25\right), \quad$ which leads to $S t_{0}$ $=\left(25 \rho_{l} d^{2} \operatorname{Re}_{0}\right) /\left(18 \rho_{G, 0} D_{J}^{2}\right)$. For the parameter values provided in Table II, and $\operatorname{Re}_{0}=500$ (the value used in the TP simulations), $S t_{0}=2.78$, which is comparable to the mean $S t_{0}$ of 3 used in the simulations of Miller and Bellan ${ }^{24}$ for temporal mixing layers. 
TABLE II. Properties used in the simulations (evaluated at atmospheric pressure and $T=350 \mathrm{~K}$ from correlations for air and decane found in Miller et al., 1998). The subscripts $L, C$, and $V$ denote the liquid, carrier gas, and evaporated vapor, respectively.

\begin{tabular}{ll}
\hline \hline Property & \multicolumn{1}{c}{ Value } \\
\hline$W_{C}$ & $28.97 \mathrm{~kg} / \mathrm{kg} \mathrm{mole}$ \\
$W_{V}$ & $142.0 \mathrm{~kg} / \mathrm{kg} \mathrm{mole}$ \\
$C_{p, C}$ & $1006.24 \mathrm{~J} /(\mathrm{kg} \mathrm{K})$ \\
$C_{p, V}$ & $1980 \mathrm{~J} /(\mathrm{kg} \mathrm{K})$ \\
$C_{L}$ & $2520.5 \mathrm{~J}(\mathrm{~kg} \mathrm{~K})$ \\
$\operatorname{Pr}_{G}$ & 0.695 \\
$\rho_{L}$ & $642 \mathrm{~kg} / \mathrm{m}^{3}$ \\
$T_{B, L}$ & $447.7 \mathrm{~K}$ \\
$h_{V}^{0}$ & $5.2 \times 10^{5} \mathrm{~J} / \mathrm{kg}$ \\
\hline \hline
\end{tabular}

\section{RESULTS}

Noncircular SP flow jets have been studied extensively numerically, and their distinctive features are already established. However, to document the differences between TP and SP jets, simulations of SP flows are here performed for the same configurations as those of the TP flow simulations, so as to allow meaningful comparisons. The finding of same qualitative features between our SP laminar simulations and results from SP turbulent jets (e.g., Miller et al. ${ }^{18}$ ) supports the credibility of the present simulations. In addition to documenting the effect of the interaction between drops and the flow through comparisons of SP and equivalent TP flow simulations, we are also interested in exploring the different drop dispersion patterns by illustrating the instantaneous Eulerian drop-number density computed by ensemble averaging over the drops

$$
n_{d}=\sum_{\alpha} \frac{w_{\alpha}}{\Delta V_{\alpha}} .
$$

The drop dispersion characteristics influence the distribution of the evaporated species, and thus gaseous mixing. To understand the drop dispersion we explore the dynamics of the flow with and without drops and identify the mechanisms responsible for the different dispersion behavior. To quantify gaseous mixing, we focus on the $Y_{V}$ distribution. All of these quantities are evaluated at the nondimensional time $t^{*}$ $=t \Delta U_{0} / D_{j}$, corresponding to the achievement of a steadystate condition based on the mass flux entering and exiting the domain, $t_{s}^{*}$ (see below). These times are listed in Table I.

\section{A. Achievement of a steady-state mass flux condition}

The achievement of a steady-state mass flux, $Q$ $=\int_{S} \rho u_{1} d x_{2} d x_{3}$ as defined in Liepmann and Gharib, ${ }^{37}$ is well known for SP jets. Illustrated in Fig. 2(a) is the nonodimensional mass-flux difference between outlet and inlet, $\left(Q-Q_{0}\right) / Q_{0}$, calculated for SP jets at several $\operatorname{Re}_{0}$ values at steady-state (see figure caption for $t^{*}$ ); we define the steadystate as that corresponding to the situation achieved when $\left(Q-Q_{0}\right) / Q_{0}$ is no longer a function of time. The value of $\left(Q-Q_{0}\right) / Q_{0}$ measures entrainment and can be used to compare different simulations. None of the uncertainties associated with the quantification of entrainment according to the
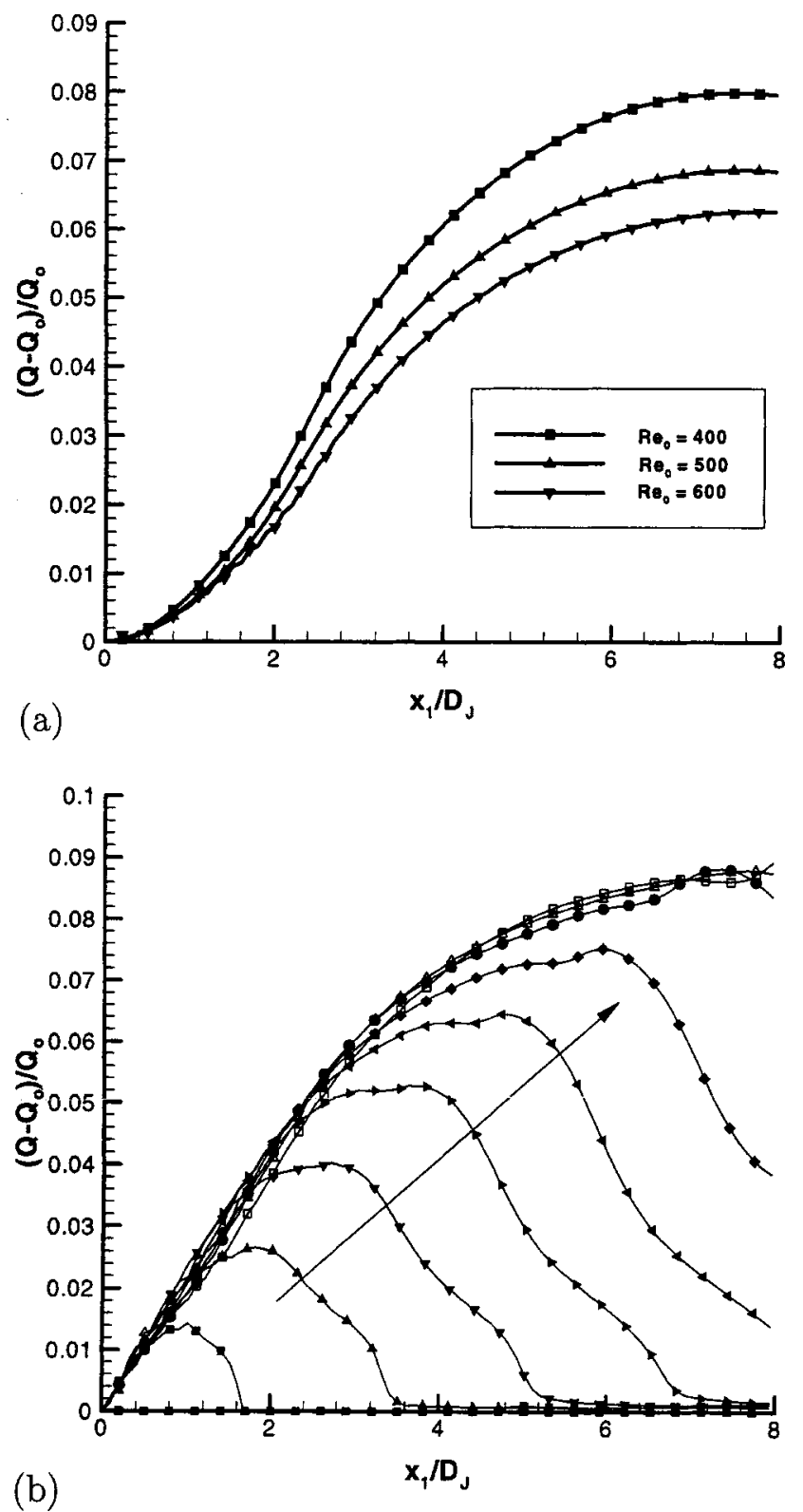

FIG. 2. Mass flux (a) steady-state profile for SP square jets having different $\operatorname{Re}_{0}\left(t^{*}=14.9,14.2\right.$, and 13.7 for $\mathrm{Re}_{0}=400,500$, and 600) and (b) development of the steady-state profile as a function of time for Run 1 TP corresponding to the simulations in Table I.

domain size, as discussed by Grinstein, ${ }^{17}$ intervene here because there is no intent to obtain agreement with a set of experimental observations; instead, the intent is to compare results from different simulations using a previously established criterion.

At steady-state, jets attain a larger value of ( $Q$ $\left.-Q_{0}\right) / Q_{0}$ with decreasing $\operatorname{Re}_{0}$, due to the larger $\mu_{G}$. Indeed, inviscid fluids do not entrain (the only mechanism for inviscid fluids to affect surrounding fluids is through pressure pushing), and entrainment increases with viscosity. The fact that TP jets also achieve a steady-state is documented in Fig. 2(b) where $\left(Q-Q_{0}\right) / Q_{0}$ is depicted as a function of $x_{1}^{*}$ $=x_{1} / D_{j}$ for a TP circular jet having $\operatorname{Re}_{0}=500$. The curves represent $\left(Q-Q_{0}\right) / Q_{0}$ at increments of 1.47 in $t^{*}$. As the jet evolves in time, $\left(Q-Q_{0}\right) / Q_{0}$ eventually becomes a mono- 

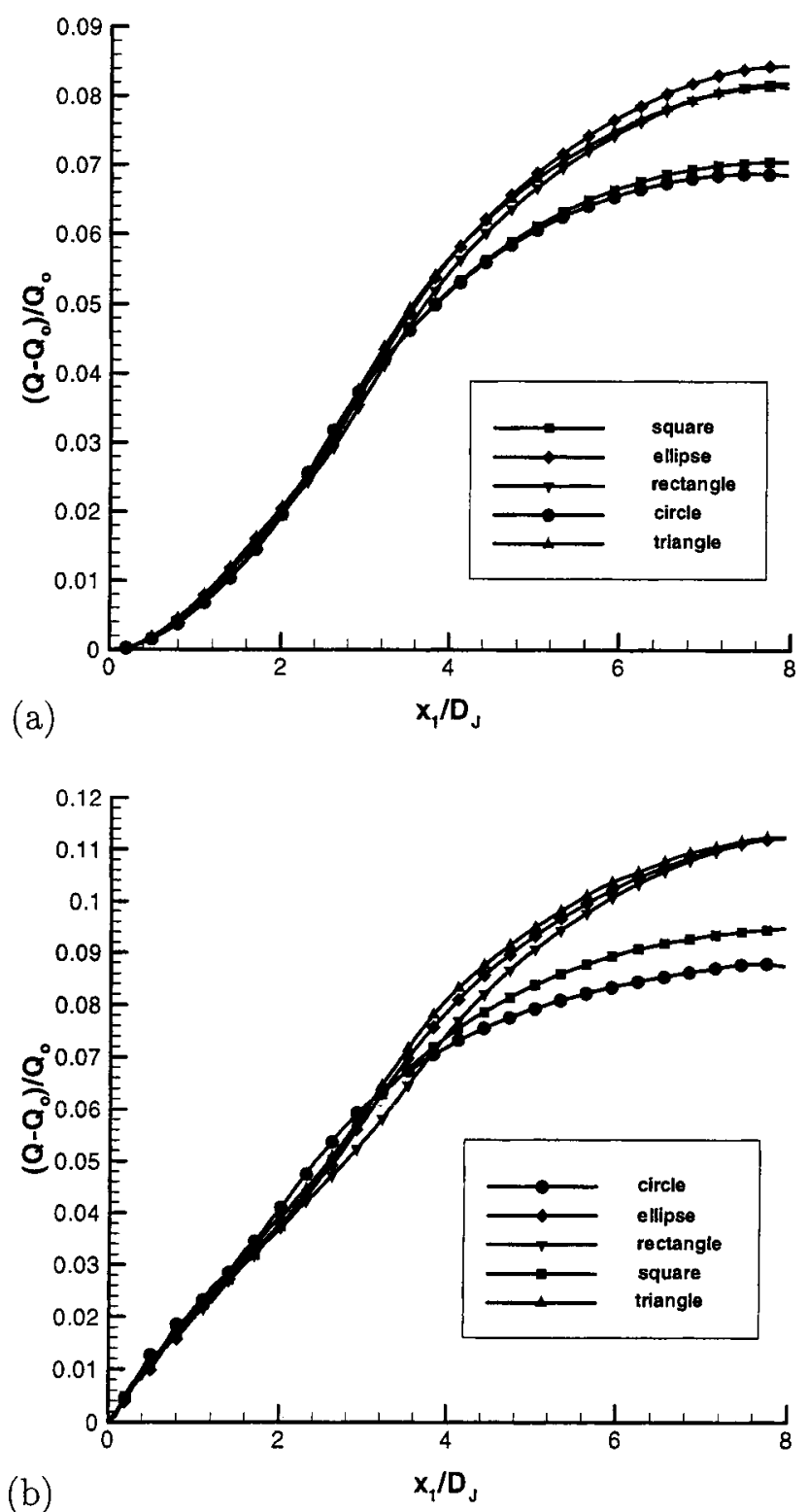

FIG. 3. Steady-state mass flux for simulations listed in Table I: (a) profiles for Runs $1 \mathrm{SP}$ to 5SP, and (b) profiles for Runs 1TP to 5TP.

tonic and increasing function of $x_{1}^{*}$ and its variation is no longer a function of $t^{*}$, clearly conforming to the definition of the steady-state.

The superior entrainment rate of the SP noncircular jets is displayed in Fig. 3(a). Whereas the square geometry is only marginally beneficial compared with the circular one, in contrast, rectangular, elliptical, and triangular jets exhibit substantial (in excess of 20\%) enhancement in entrainment. The failure of the square jet to produce similar enhancement is attributed to its lack of axis switching ${ }^{18}$ despite the rotation of its axis by $45^{\circ}$; this rotation plays a significant role when the flow is turbulent, but it is not as important for laminar flows such as the one under consideration herein. Parallel plots for the TP flow simulations are shown in Fig. 3(b). The entrainment in TP flows is enhanced over that of SP flows by more than $30 \%$. While this result could partly be attributable to the increase in the injected mass, it will be shown below that there are detailed aspects of the drop-gas interaction that are the primary contributors to this effect. In fact, it is shown below that there are different topological features that develop in TP jets that cannot be explained by the mass addition; instead, they are attributed to the drop-gas interaction. All further aspects investigated herein involve examination of the steady-state database.

\section{B. Vorticity evolution}

To elucidate some of the differences between SP and TP jets, we focus on the vorticity aspect of the flow. Vorticity is of interest because it has been linked to entrainment rates ${ }^{15}$ as the increase in the mass flux of rotational fluid is one of the mechanisms through which fluid may be entrained. Moreover, for circular jets, the near-field streamwise vorticity has been shown to play a central role in generating $3 \mathrm{D}$ structures and also in sustaining entrainment from the surrounding of the jet, thereby promoting its growth. ${ }^{37}$ Furthermore, it has also been observed ${ }^{37}$ that as the flow moves downstream, the streamwise vorticity becomes more important than the azimuthal one, and that past the potential core regime, it controls the entrainment process. To evaluate the vorticity characteristics of the jets, consider the vorticity equation for TP flow derived from Eqs. (1) and (2):

$$
\begin{aligned}
\frac{D \boldsymbol{\omega}}{D t}= & (\boldsymbol{\omega} . \nabla) \mathbf{u}-(\nabla . \mathbf{u}) \boldsymbol{\omega}-\nabla\left(\frac{1}{\rho}\right) \times \nabla p+\nabla \times\left(\frac{1}{\rho} \nabla . \overline{\bar{\tau}}\right) \\
& +\nabla \times\left(\frac{1}{\rho} \mathbf{S}_{I I}\right)-\nabla \times\left(\frac{1}{\rho} S_{I} \mathbf{u}\right),
\end{aligned}
$$

where $D / D t$ is the substantial derivative, and of the vorticity magnitude squared equation

$$
\begin{aligned}
\frac{D(\boldsymbol{\omega} \cdot \boldsymbol{\omega})}{D t}= & 2 \boldsymbol{\omega} \cdot(\boldsymbol{\omega} \cdot \nabla \mathbf{u})-2(\nabla \cdot \mathbf{u}) \omega^{2} \\
& -2 \boldsymbol{\omega} \cdot\left[\nabla\left(\frac{1}{\rho}\right) \times \nabla p\right]+2 \boldsymbol{\omega} \cdot\left[\nabla \times\left(\frac{1}{\rho} \nabla \cdot \overline{\bar{\tau}}\right)\right] \\
& +2 \boldsymbol{\omega} \cdot\left[\nabla \times\left(\frac{1}{\rho} \mathbf{S}_{I I}\right)\right]-2 \boldsymbol{\omega} \cdot\left[\nabla \times\left(\frac{1}{\rho} S_{I} \mathbf{u}\right)\right] .
\end{aligned}
$$

The first term in the above equations represents the stretching and tilting contribution, the second term describes the effect of dilatation, the third term is the baroclinic participation to vorticity production, the fourth term portrays the viscous contribution, the fifth term represents the momentum source due both to drag and evaporation, and the last term describes the contribution of the evaporated mass to vorticity production. For SP flows, the last two terms are null.

\section{Single-phase versus two-phase jets}

Considering the finding of Liepmann and Gharib ${ }^{37}$ regarding the crucial role of the streamwise vorticity in jet entrainment, we display in Figs. 4(a) and 4(b) the nondimensional streamwise $\left(x_{1}, x_{3}\right)$-plane-average, both average and rms vorticity budgets, $\omega_{1}^{\text {ave }}$ and $\omega_{1}^{\text {rms }}$, for the SP circular jet. Most of the generated $\omega_{1}^{\text {ave }}$ is due to the action of the stretch- 


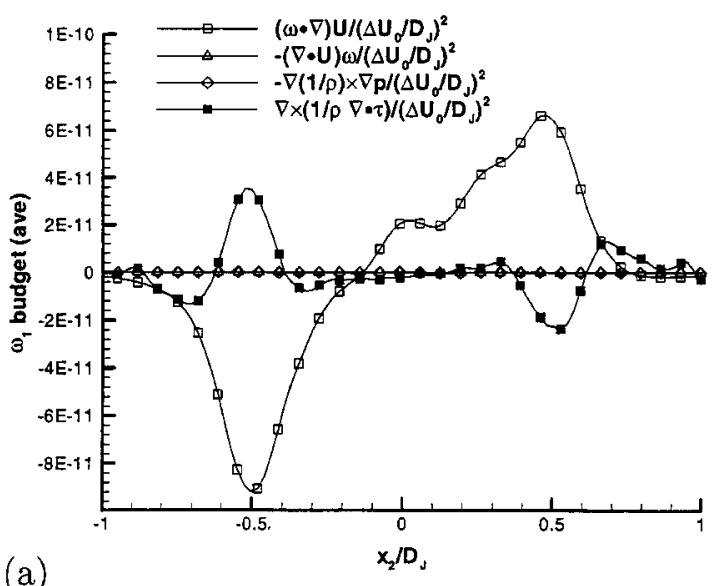

(a)

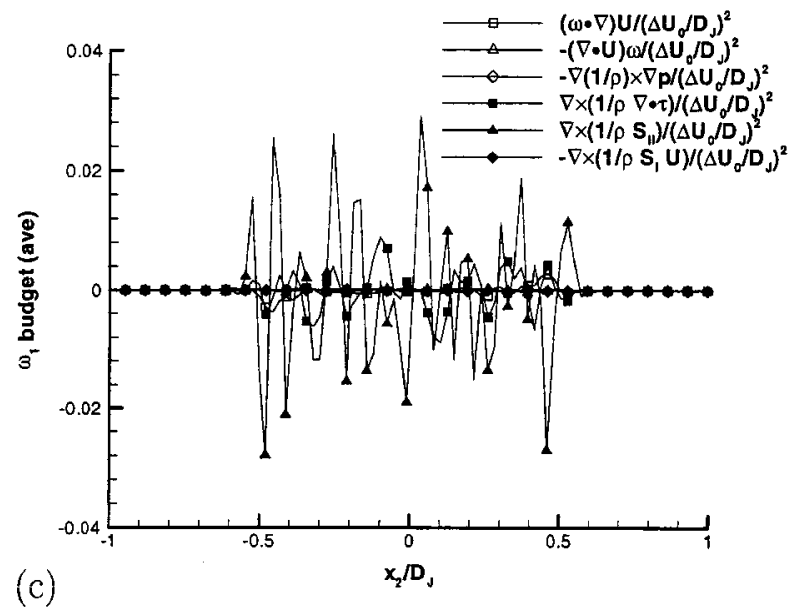

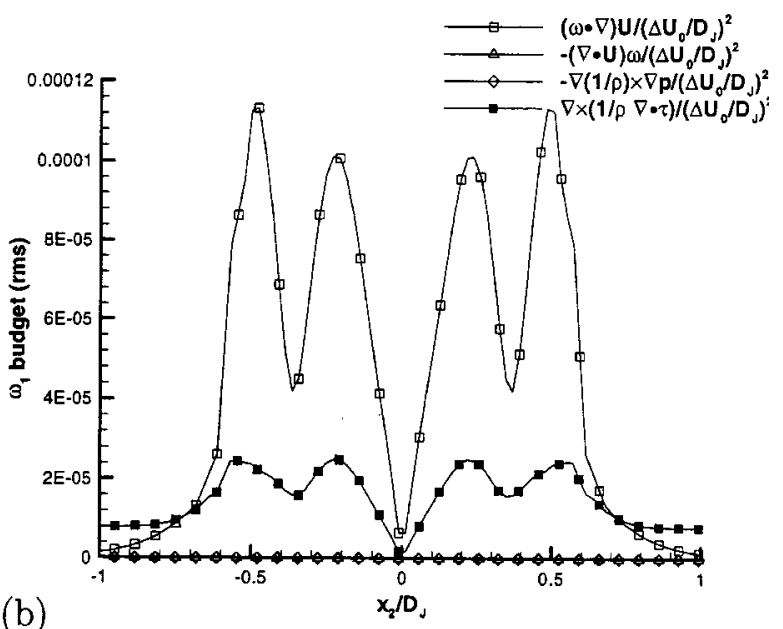

(b)

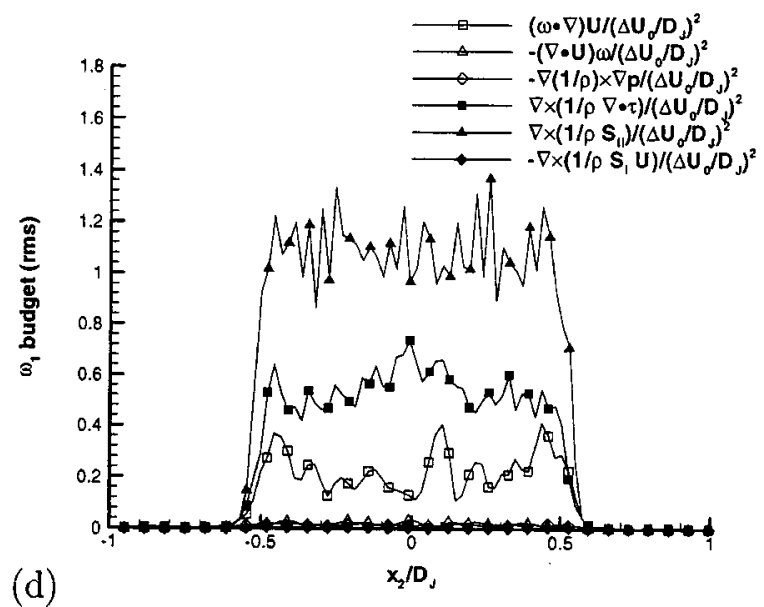

FIG. 4. Streamwise vorticity at steady-state entrainment for circular cross-section jets (Runs 1SP and 1TP of Table I): (a) plane average for Run 1SP, (b) rms for Run 1SP, (c) plane average for Run 1TP, and (d) rms for Run 1TP.

ing and tilting term, which is responsible for the creation of 3D structures, while viscosity partly counteracts its effects. Examining $\omega_{1}^{\text {rms }}$, we identify, similar to $\omega_{1}^{\text {ave }}$, the important role of the stretching and tilting action in producing fluctuations, and also note the secondary role of the viscous contribution. The minute value of $\omega_{1}^{\text {ave }}$ and small value of $\omega_{1}^{\text {rms }}$ indicate that the jet is still laminar. In comparison, the TP jet, while still generating a small amount of $\omega_{1}$, produces a factor of $10^{8}$ more $\omega_{1}^{\text {ave }}$ [see Fig. 4(c)] and a factor of $10^{4}$ more $\omega_{1}^{\text {rms }}$ [see Fig. 4(d)]; the finding that the drop presence increases the vorticity and the fluctuations in the flow is in agreement with the experimental observations of McDonell and Samuelsen. ${ }^{38}$ The momentum source term is the dominant contribution to both $\omega_{1}^{\text {ave }}$ and $\omega_{1}^{\text {rms }}$, followed by the viscous term; a minor production of $\omega_{1}^{\text {rms }}$ is due to the stretching and tilting term. Therefore, it is apparent that the important mechanism of $\omega_{1}$ creation that enhances entrainment is considerably stronger in TP circular jets than in their SP counterpart, and is due to the gas-drop interaction.

A comparison of the $\omega_{1}$ produced by all injection geometries for SP jets (not shown) reveals that $\omega_{1}^{\text {ave }}$ for the triangular jet is seven orders of magnitude larger than those of all other jets; this is attributed to the special corner configuration whose features were discussed by Koshigoe et al. ${ }^{13}$ Their finding that the vertices of the triangle were producing predominantly small-scale structures is consistent with the enhanced $\omega_{1}$ production found herein. Koshigoe et al. ${ }^{13}$ determined that there is a differentiation between coherent structures, which were overwhelmingly formed only at the flat sides, and turbulent flow with predominantly small scales found at the triangle vertices. For rectangular jets, Tam and Thies ${ }^{16}$ found that the corner instability modes quickly induced significant mixing, thereby effectively rounding off the corners of the jet and emulating the more circular geometry in the downstream direction. Of all geometries, the circular one produces the smallest $\omega_{1}^{\text {rms }}$, by about one order of magnitude; this indicates that the circular geometry is the least effective at producing the fluctuations necessary for enhancing entrainment and inducing good mixing; this result is consistent with the findings of Husain and Hussain. ${ }^{19}$ As discussed below, the situation is considerably different for TP jets, where the magnitude of both $\omega_{1}^{\text {ave }}$ and $\omega_{1}^{\text {rms }}$ is similar for all configurations. The reason for this discrepancy between SP and TP jets is partly due to the fact that the primary contribution to the $\omega_{1}$ production for SP jets is from the stretching and tilting term, which is geometry dependent, while the essential contribution for TP jets is from the momentum-source term (due both to drag and evaporation), 


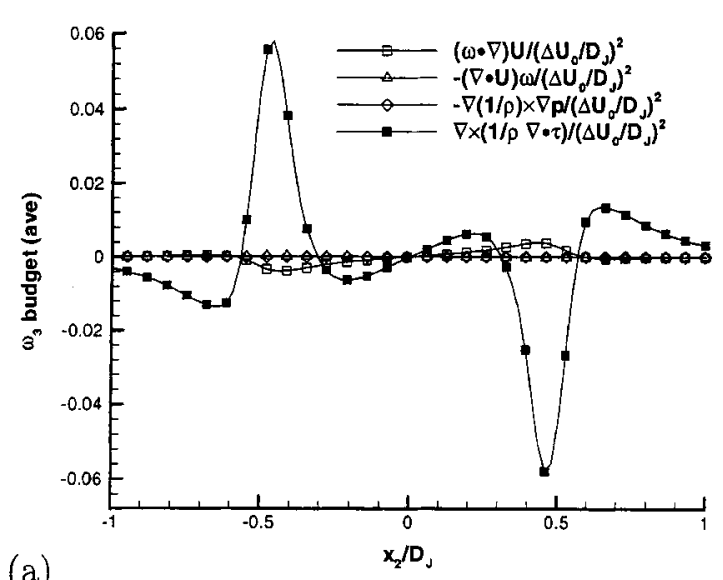

(a)

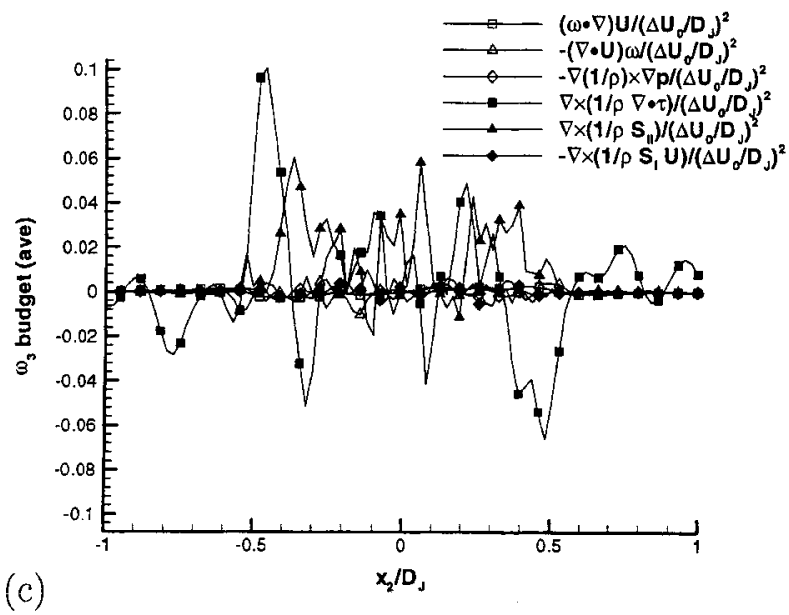

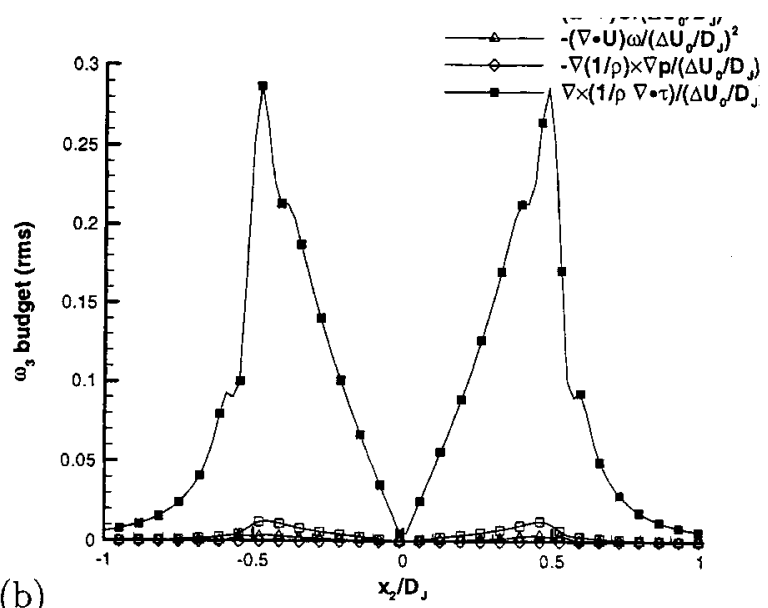

(b)

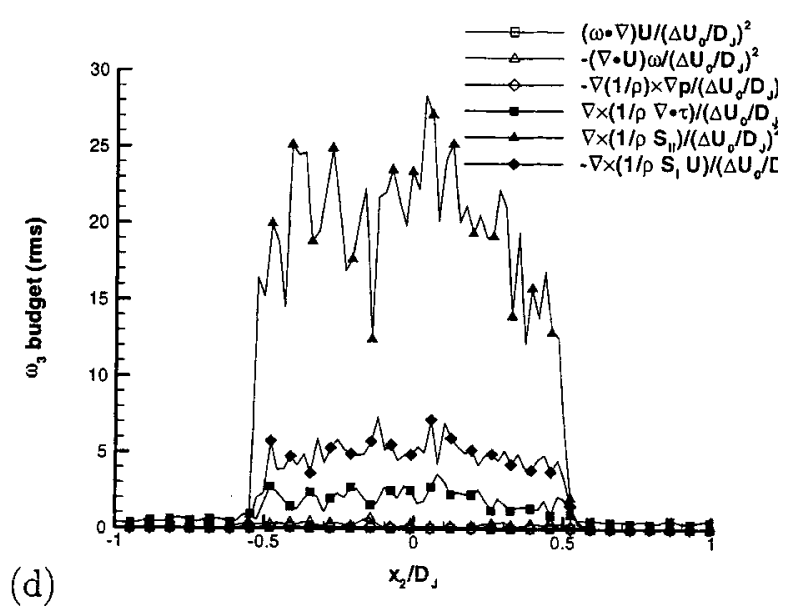

FIG. 5. Spanwise vorticity at steady-state entrainment for circular cross-section jets (Runs 1SP and 1TP of Table I): (a) plane average for Run 1SP, (b) rms for Run 1SP, (c) plane average for Run 1TP, and (d) rms for Run 1TP.

which is related to the drop number and size that are initially the same in all simulations. This result indicates that one should use caution in extrapolating SP results into the TP setting, and also implies that in the TP setting there are effects tending to offset the benefit of the corner configuration; these effects are discussed below in the context of drop dispersion and mixing.

Depicted in Figs. 5(a) and 5(b) are the nondimensional spanwise plane-average and rms vorticity budgets, $\omega_{3}^{\text {ave }}$ and $\omega_{3}^{\text {rms }}$, for the SP circular jet. Equivalent plots for the TP flow are illustrated in Figs. 5(c) and 5(d). For the SP jet, the dominant contribution to both $\omega_{3}^{\text {ave }}$ and $\omega_{3}^{\text {rms }}$ is from viscous effects, with a minute contribution from the stretching and tilting term; all other terms are negligible. For the TP jet, the momentum-source term rivals the viscous term in contributing to $\omega_{3}^{\text {ave }}$, whereas $\omega_{3}^{\text {rms }}$ is clearly dominated by the momentum-source terms, with the mass source being next in order of importance and finally the viscous term being almost a factor of 10 smaller than the leading contribution. Noteworthy, $\omega_{3}^{\text {ave }}$ for the TP jet is about $60 \%$ larger than that for the SP counterpart, and furthermore, $\omega_{3}^{\text {rms }}$ is a factor of $10^{2}$ larger than that for the SP jet.

To complete the vorticity budget analysis, vorticitymagnitude plots are displayed in Figs. 6(a)-6(d). While viscosity is mainly responsible for the production of $\boldsymbol{\omega} \cdot \boldsymbol{\omega}$ for the SP jet, the momentum-source term, and to a much lesser extent the mass-source term as well as viscous effects contribute to the creation of $\boldsymbol{\omega} \cdot \boldsymbol{\omega}$ for the TP jet. Consistent with the vorticity budget discussed above, $(\boldsymbol{\omega} \cdot \boldsymbol{\omega})^{\text {ave }}$ is a factor of 5 , and $(\boldsymbol{\omega} \cdot \boldsymbol{\omega})^{\mathrm{rms}}$ is a factor of $10^{2}$ larger for the TP jet. Noteworthy, these ratios between SP and TP $\boldsymbol{\omega} \cdot \boldsymbol{\omega}$ magnitudes are though smaller than the corresponding ones for $\omega_{1}$, and closer to the equivalent ratios for $\omega_{3}$.

The enhancement of $\omega_{1}, \omega_{3}$ and $\boldsymbol{\omega} \cdot \boldsymbol{\omega}$ in TP jets with respect to SP jets is of interest for understanding the nature of the difference between the two situations. Based on plots similar to those of Figs. 4 to 6 (not shown), it is clear that the triangular cross section produces most $\omega_{3}$ and $\boldsymbol{\omega} \cdot \boldsymbol{\omega}$ enhancement through the addition of evaporating liquid drops to the flow, although as discussed above the enhancement in $\omega_{1}$ is the smallest. In the next section we discuss how these vorticity effects influence drop dispersion and species mixing.

The vorticity analysis shows that it is the interaction between the drops and the gas, rather than the added liquid mass to the gaseous jet, which increases the total mass of the jet, that is mostly responsible for the increased entrainment of the TP circular jet compared to the SP one. In particular, for the present situation it is the drag term rather than the evaporation term that produces most of the vorticity from momentum interaction. This result follows from the fact 


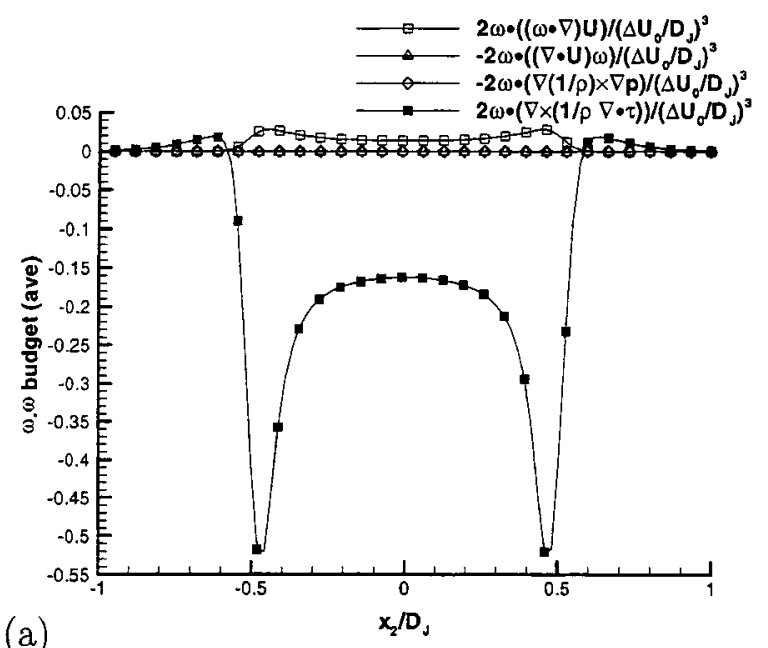

(a)

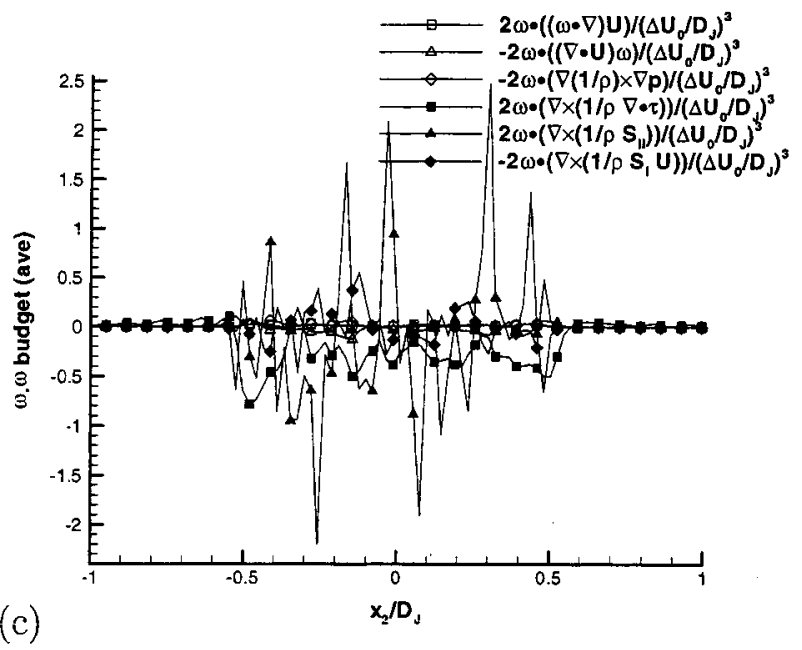

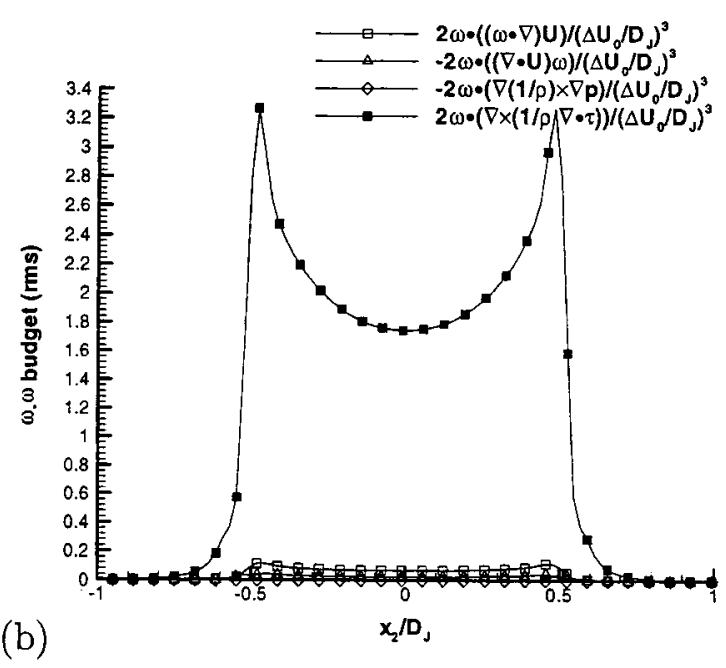

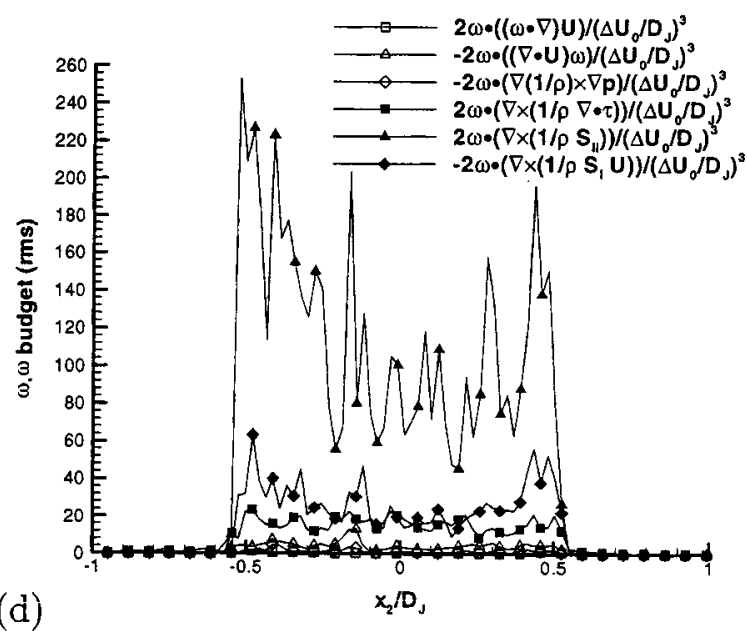

FIG. 6. Vorticity square at steady-state entrainment for circular cross-section jets (Runs 1SP and 1TP of Table I): (a) plane average for Run 1SP, (b) rms for Run 1SP, (c) plane average for Run 1TP, and (d) rms for Run 1TP.

that initially $\|\mathbf{v}\|<\|\mathbf{u}\|$, and therefore $\nabla \times\left((1 / \rho) S_{I} \mathbf{u}\right)$ represents a maximum for the evaporation contribution to $\nabla \times\left((1 / \rho) \mathbf{S}_{I I}\right)$. This implies that $\nabla \times\left((1 / \rho) \mathbf{S}_{I I}\right)$ $-\nabla \times\left((1 / \rho) S_{I} \mathbf{u}\right)$ represents a minimum for the drag term contribution to $\nabla \times\left((1 / \rho) \mathbf{S}_{I I}\right)$. The fact that this minimum of the drag term is larger than the maximum of the evaporation term contribution to $\nabla \times\left((1 / \rho) \mathbf{S}_{I I}\right)$ is easily observed in Figs. 4-8. Under other conditions (e.g., larger $T_{G, 0}$, larger initial drop velocity compared to the gas velocity), it is possible that the evaporation term will play a larger role in the momentum interaction. The finding that in TP jets the dropgas interaction is the primary phenomenon creating vorticity, and that the vorticity magnitude is considerably larger than in SP jets, indicates that a transitional/turbulent state will be considerably easier to reach for the TP jet; the momentumsource term representing the interaction between drops and gas will play the major role in achieving transition, followed by the mass-source term.

The reasons for the enhanced entrainment of TP jets with noncircular cross sections compared to the circular one is addressed next.

\section{Two-phase jets with different cross-section geometry}

Comparisons between $\omega_{1}, \omega_{3}$ and $\boldsymbol{\omega} \cdot \boldsymbol{\omega}$ budgets for TP elliptic, square, and rectangular jets with the circular jet reveal that neither the plane average nor the rms magnitude is considerably larger in the former than in the latter. As an example, illustrated in Fig. 7 are $\omega_{1}^{\mathrm{rms}}, \omega_{3}^{\mathrm{rms}}$ and $(\boldsymbol{\omega} \cdot \boldsymbol{\omega})^{\mathrm{rms}}$ for the elliptic jet to be directly compared with Figs. 4(d), 5(d), and 6(d). However, the triangular jet exhibits much larger $\omega_{1}^{\mathrm{rms}}, \omega_{3}^{\mathrm{rms}}$, and $(\boldsymbol{\omega} \cdot \boldsymbol{\omega})^{\mathrm{rms}}$ peaks at its $x_{2} / D_{J}=0$ vertex (see Fig. 8), providing clear evidence of fine-scale formation whose main contribution is no longer from the stretching and tilting effect as in the SP case, but from the momentum interaction with the drops.

Examination of the vorticity plots leads to the conclusion that, with the exception of the triangular geometry, the difference between the circular and noncircular jets is in the enhanced absolute spreading of the rms profiles for these latter. A simple estimate of the ratio of the inlet to $\left(x_{1}, x_{3}\right)$-plane-average spread shows that the triangular jet 


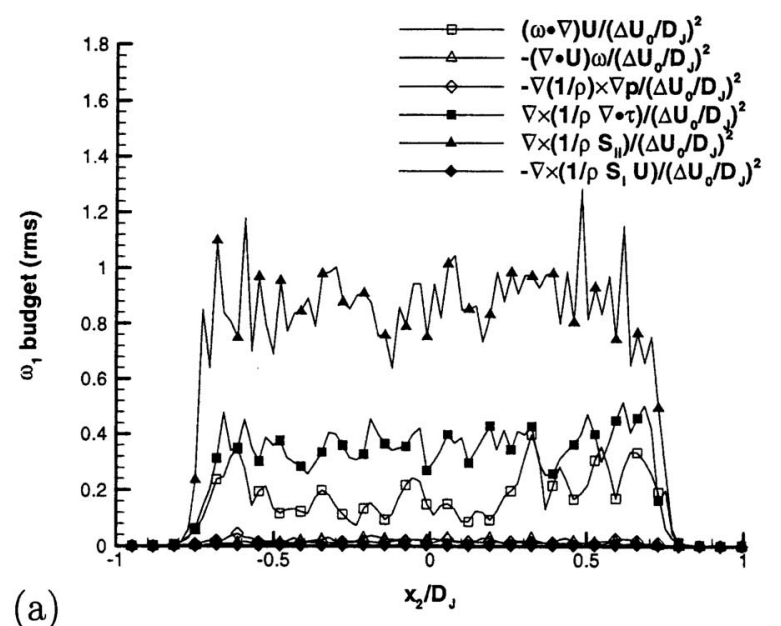

(a)

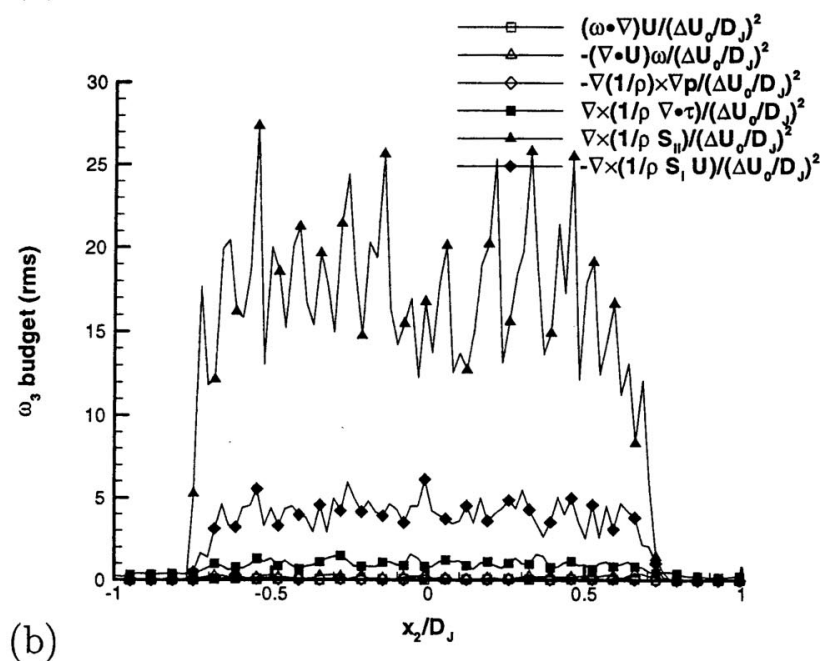

(b)

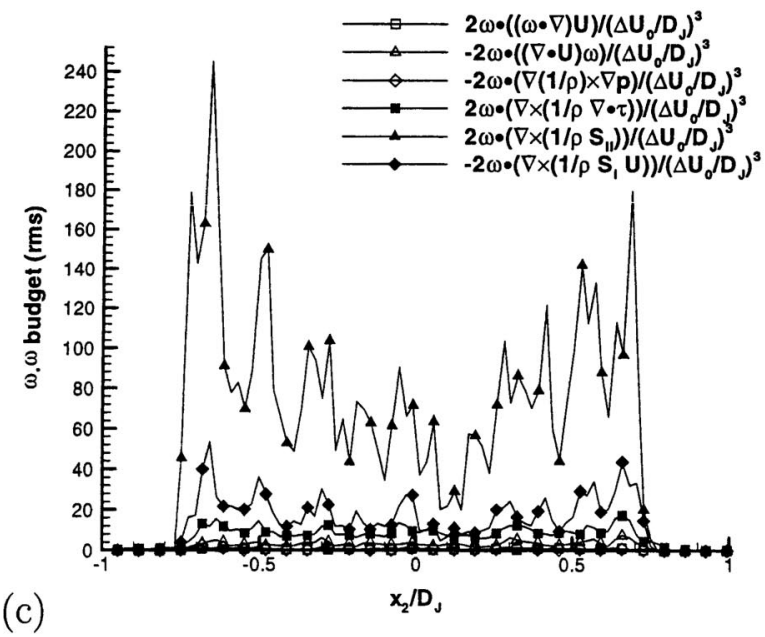

FIG. 7. Streamwise vorticity rms (a), spanwise vorticity rms (b), and vorticity square rms (c) at steady-state for the run $2 \mathrm{TP}$.

spreads relatively twice as much as all other jets whose ratio is similar.

\section{Velocity distributions and axis switching}

The potential core of a jet is here defined as the region beyond which the velocity no longer equals that at the inlet. In $3 \mathrm{D}$, this region is delineated by a surface. To understand
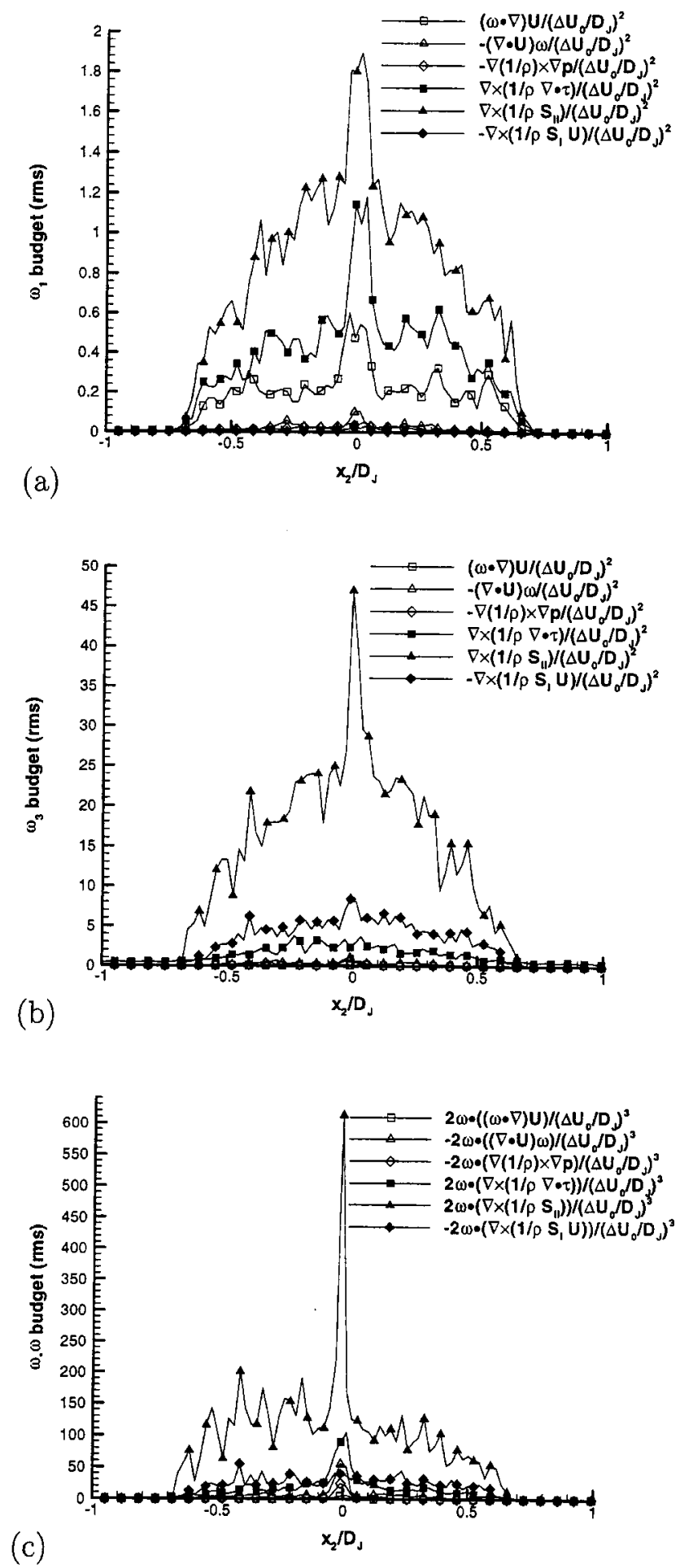

FIG. 8. Streamwise vorticity rms (a), spanwise vorticity rms (b), and vorticity square rms (c) at steady-state for the run 5TP.

the impact of the drops in altering the gas-velocity profile, we display in Fig. 9 the potential cores for SP and TP jets at $x_{2} / D_{J}=0$. The potential core of noncircular gaseous jets is shorter than that of circular jets, with the square jet being closer to the circular jet and the rectangular one being the shortest. We note that the potential core is reduced both in the $x_{1}$ and $x_{3}$ directions, and that for the triangular configuration there is an asymmetry with respect to $x_{3}=0$, as ex- 

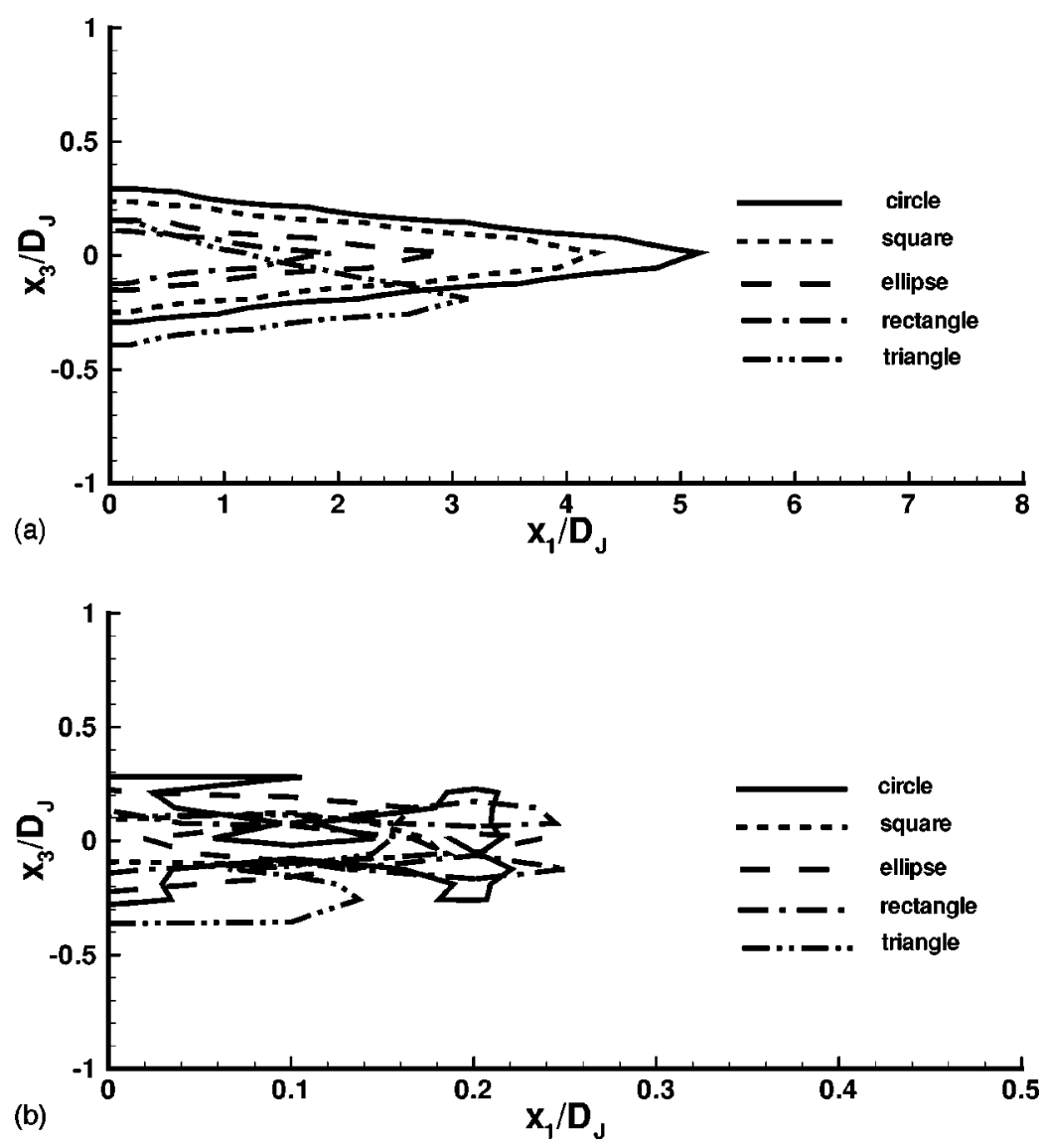

FIG. 9. Potential cores at $x_{2} / D_{J}=0$ for SP (a) and TP (b) jets, all at steady state. pected. The result of the drop interaction with the flow is to reduce the potential core by about an order of magnitude in the streamwise direction. Moreover, it is also clear that the increased vorticity for the TP jets induces local fluctuations in the velocity (in agreement with the experimental findings of McDonell and Samuelsen ${ }^{38}$ ), typically yielding jagged and very asymmetric core profiles; however, all cores seem to be approximately of the same length.

To further investigate the features of the gas velocity that are altered when introducing evaporating drops, we first document in Fig. 10 the well-known process of axis switching. The plots of Fig. 10 are for the SP elliptic jet, showing the velocity vectors at stations $x_{1} / D_{J}=0,2.5,5.0$, and 7.5. In these figures, the jet core is delineated by the region having close-to-null velocity. Due to an initial disproportionate increase in spreading in the minor axis direction with respect to that in the major axis, the jet eventually switches axis; these results are in qualitative agreement with similar findings (e.g., Miller et al. ${ }^{18}$ ) for turbulent SP jets. Although the computational domain is not long enough to capture the ultimate axis switching, it is evident that by $x_{1} / D_{J}=7.5$ the initial ellipse resembles a circle; also, the velocity vectors are such that further extension in the $x_{3}$ direction is anticipated. Parallel plots of the TP elliptic jet are depicted in Fig. 11 at the same locations as those in Fig. 10. The velocity vectors in the $x_{1} / D_{J}=0$ plane show a large irregularity that is typical of two-phase flow inlet conditions in which the drops are randomly injected (see McDonell and Samuelsen ${ }^{38}$ ). By $x_{1} / D_{J}=2.5$, the TP profiles closely resemble the SP profiles, with subtle differences. Departures from the SP profiles are, however, evident by $x_{1} / D_{J}=5$, and the symmetry with respect to $x_{3}=0$ is broken. By $x_{1} / D_{J}=7.5$, a kidney shaped complex circulation zone becomes established. Such increase in asymmetry with respect to top and bottom parts of a jet was experimentally observed by Liepmann and Gharib ${ }^{37}$ in high Re spatial circular jet experiments and was attributed to distortions associated with the development of the flow past the location of the potential core. In those experiments, initial instabilities and further flow development triggered additional instabilities leading to asymmetry past the potential core where streamwise vorticity becomes increasingly more important than spanwise vorticity. Here, although instabilities are not intentionally imposed, the drop injection produces instabilities [see Fig. 11(a)]. Even though the flow is not turbulent and therefore the physics associated with the experiments of Liepmann and Gharib ${ }^{37}$ is not exactly applicable, the general trends are still valid in that increasing streamwise vorticity may create asymmetry. Essentially, the snapshot at $x_{1} / D_{J}=7.5$ corresponds to a location close to the outer limit of the jet core for SP jets, but to a much more downstream location with respect to the core for TP jets. In Fig. 12 we document the velocity vector distribution at $x_{1} / D_{J}=7.5$ for both SP and TP jets corresponding to circular, rectangular, square, and triangular jets. In each case, the complexity of the TP patterns should be contrasted to the much simpler well-known SP behavior. For the SP rectangular and triangular jets, the axis switching phenomenon is evident, in contrast to the SP square jet, which does not exhibit 

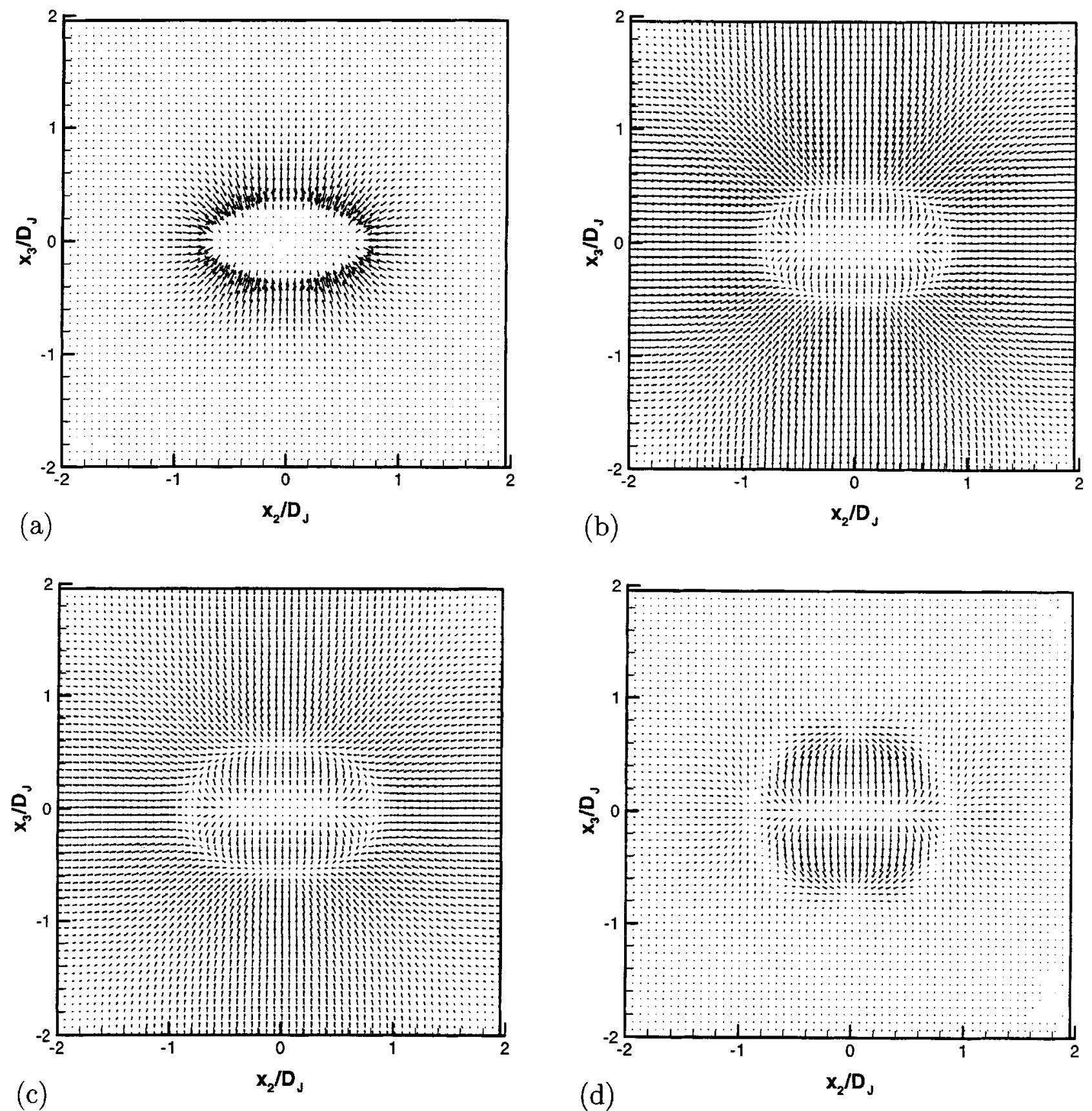

FIG. 10. Velocity vectors for the SP elliptic jet at $x_{1} / D_{J}=0$ (a); 2.5 (b); 5.0 (c); and 7.5 (d) at steady-state entrainment.

axis switching but only a $45^{\circ}$ rotation; these results are in qualitative agreement with similar findings (e.g., Miller et $a l .{ }^{18}$ ) for turbulent SP jets. The TP circular jet displays symmetry with respect to $x_{3}=0$, indicating that the lack of velocity vector symmetry associated with noncircular TP jets is a result of the initial lack of symmetry and/or corners. In contrast to the symmetric TP circular jet, the elliptic and rectangular jets display a lack of $x_{3}$ symmetry, with the lower part of the jet entraining the gas around it, and the upper part of the jet detraining gas from the jet; however, both jets retain symmetry with respect to $x_{2}$. The TP square and triangular jets exhibit even more complex patterns than the elliptic and rectangular jets, with complete asymmetry with respect to the two axes and a circulatory pattern indicating a highly 3D motion. This finding is consistent with that regarding the streamwise-vorticity production.

\section{Drop dispersion and mixing in jets with different cross-section geometry}

Since the entrainment of noncircular TP jets is superior to that of circular jets, and the velocity displays increasing asymmetry with downstream position, it is of interest to examine how this affects drop dispersion and gaseous species 

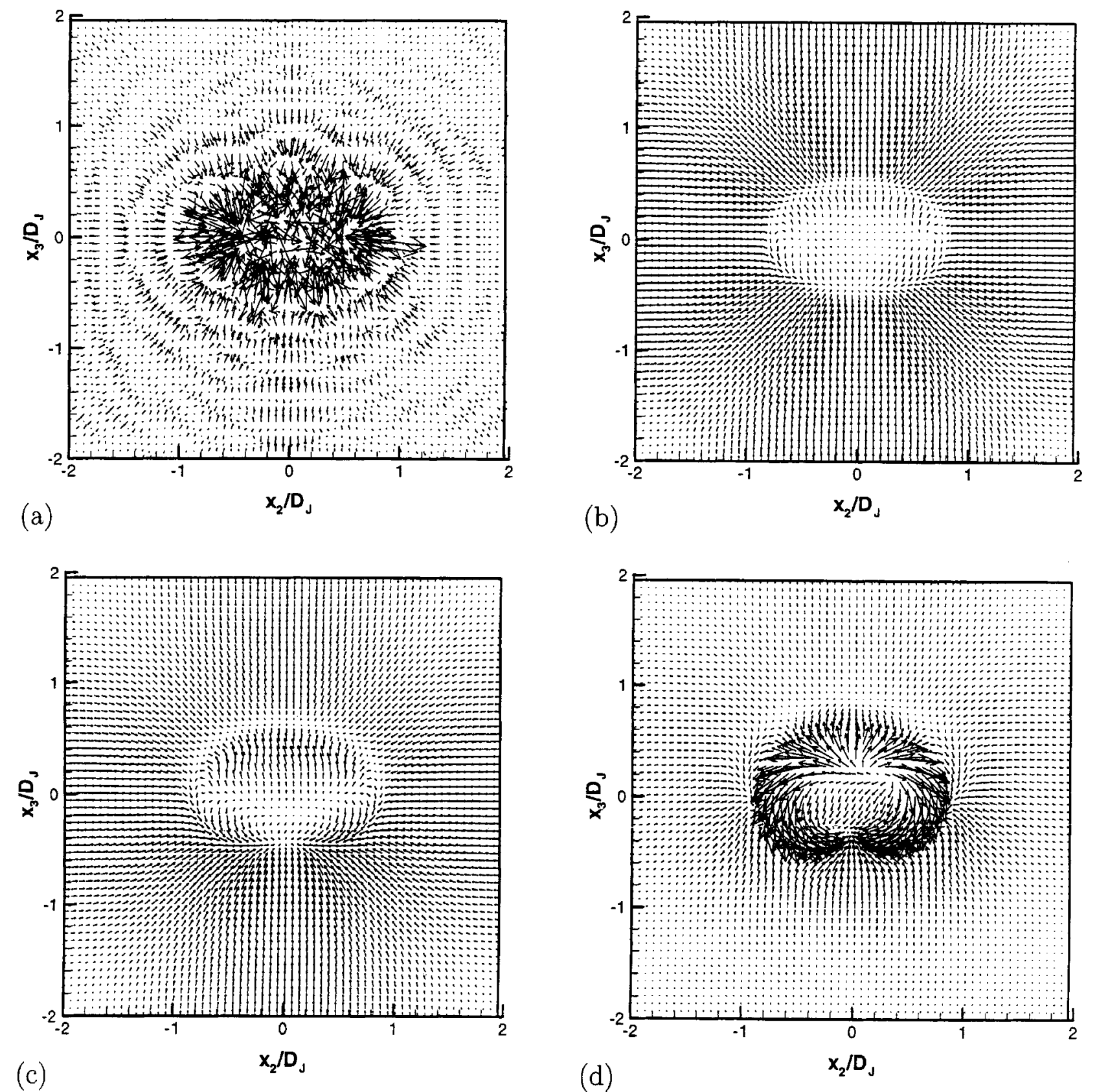

FIG. 11. Velocity vectors for the TP elliptic jet at $x_{1} / D_{J}=0$ (a); 2.5 (b); 5.0 (c); and 7.5 (d) at steady-state entrainment.

mixing. To this end, illustrated in Fig. 13 is the drop number density, $n_{d}$ [see Eq. (38)], distribution at $x_{2} / D_{J}=0$ for all cross section jets. While the information in Fig. 13 pertains only to drop location, the distribution of liquid mass per unit volume, $M_{d}=(\pi / 6) n_{d} d^{3} \rho_{L}$, depicted in Fig. 14 portrays the state of drop evaporation in the same plane.

In the context of sprays used in power plants, the circular and elliptic jets display the most attractive attributes of all configurations, in that they have the smallest $n_{d}$, an aspect that is potentially beneficial to evaporation, combustion, and low pollutant formation. The highest $n_{d}$ regions are exhibited by the triangular jet in the region of one of its vertices, as the plane at $x_{2} / D_{J}=0$ passes through it. The fine-scale formation occurring in that region (see Fig. 8) is not effective enough at dispersing the drops, which must be done through the intermediary of coherent vortices; we recall that Koshigoe et al. ${ }^{13}$ found that the coherent large scale structures form predominantly at the flat side of the triangular jet. The square and rectangular jets are intermediary between the elliptic and the triangular jet in terms of drop dispersion. The conclusion is that corner-containing cross sections tend to induce locally high concentrations of drops in laminar jets. Illustrated in Fig. 14 are the equivalent liquid-mass distributions, showing that the trends seen for $n_{d}$ are directly translatable to the liquid mass. The best configuration is that of the elliptic jet, in that the mass distribution is the most diluted; the jet core contains widespread regions of minimal 


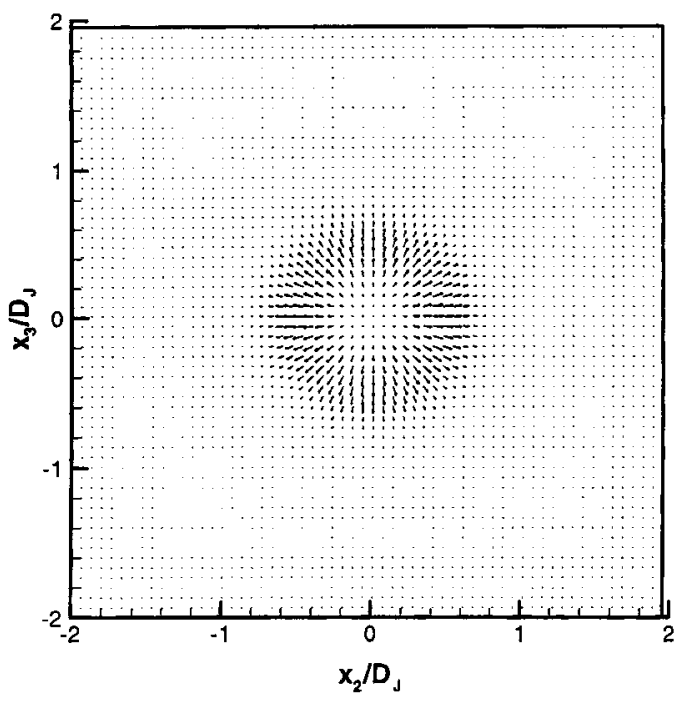

(a)

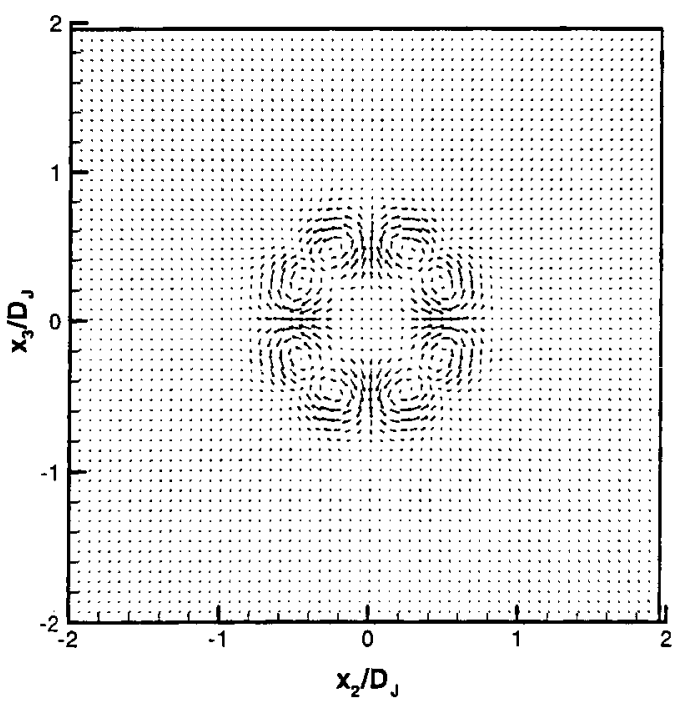

(c)

FIG. 12. Velocity vectors for SP and TP jets at $x_{1} / D_{J}=7.5$ (a) SP circular, (b) TP circular, (c) SP square, (d) TP square, (e) SP rectangular, (f) TP rectangular, (g) SP triangular, and (h) TP triangular. All at steady-state entrainment.

liquid mass, indicating that the efficiency of evaporation is high.

The illustrations in Fig. 13 allow the conservative verification of the dilute TP-flow assumption underlying the mathematical derivation. Based on the domain size, the number of grid points and the largest value of $n_{d}, 3$ $\times 10^{10} \mathrm{~m}^{-3}$, one computes that there are less than four drops per computational cell. Using the conservatively large drop volume represented by the initial condition, the percentage volume occupied by the drops in a cell is $<2 \times 10^{-3}$. In fact, this number is smaller during the entire calculation because regions of high-magnitude $n_{d}$ develop only after the drop organizational structure is established (drops congregate in regions of low vorticity and high strain) at which time the drop size has decreased considerably.

Finally, to examine how the evaporated mass is dispersed and mixes with the surrounding air, $Y_{V}$ is illustrated in Fig. 15 for the same configurations in the identical plane

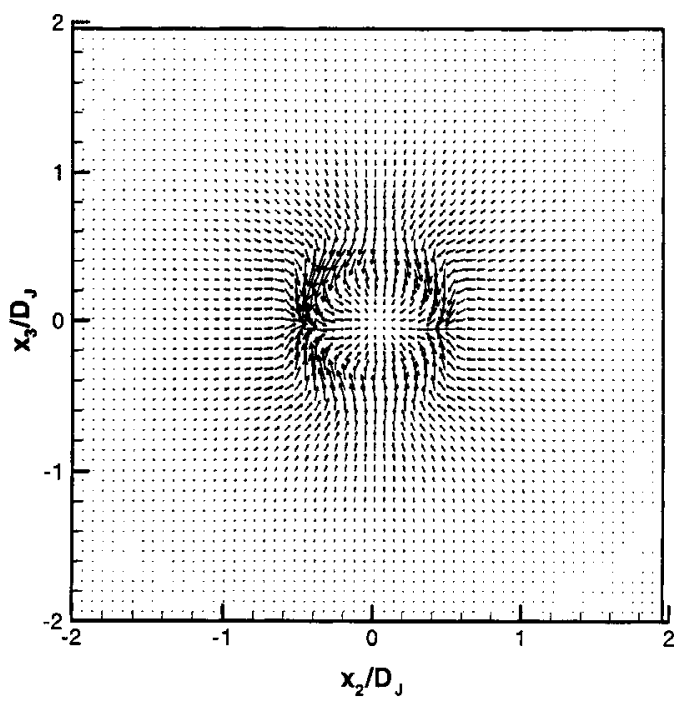

(b)

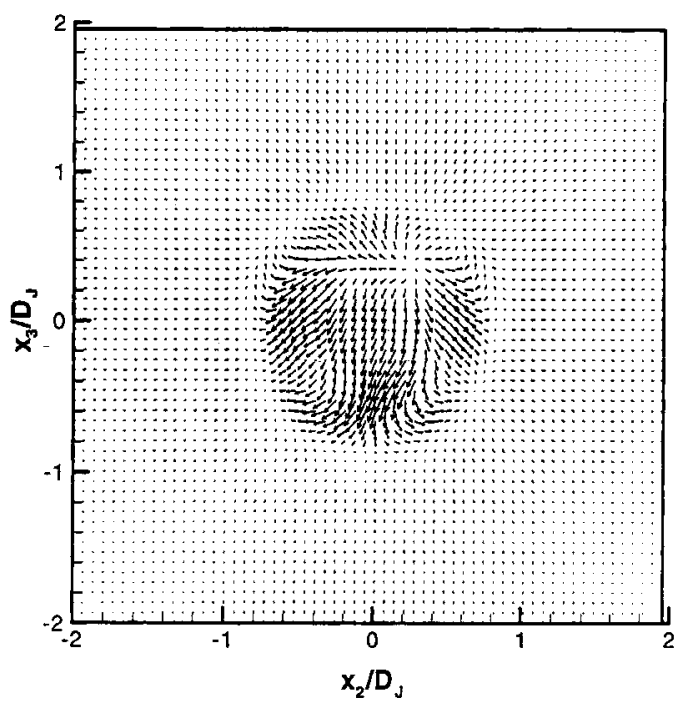

(d)

as $n_{d}$ and $M_{d}$. The circular jet contains the largest core of high evaporated-species mass fraction, followed by the triangular, the square, and finally by the elliptic and rectangular jets. The triangular jet exhibits the greatest extent in the $x_{3}$ direction, followed by the circular, square, and finally by the elliptic and rectangular jets.

Therefore, it is clear that, depending on the application, a different injection cross section might be the optimal one. The triangular and square geometries appear to both induce regions of high $n_{d}$ and have larger cores of unmixed evaporated species. Among the corner containing geometries, the rectangular one is the best suited for gaseous mixing, however, it tends to induce drop (and liquid mass) accumulation at corners. Elliptic jets are more suitable than circular ones in that all their characteristics tend to induce better drop and liquid-mass dispersion, as well as species mixing. The elliptic geometry tends to combine the small $n_{d}$ and small liquidmass aspect with good mixing characteristics, therefore mak- 

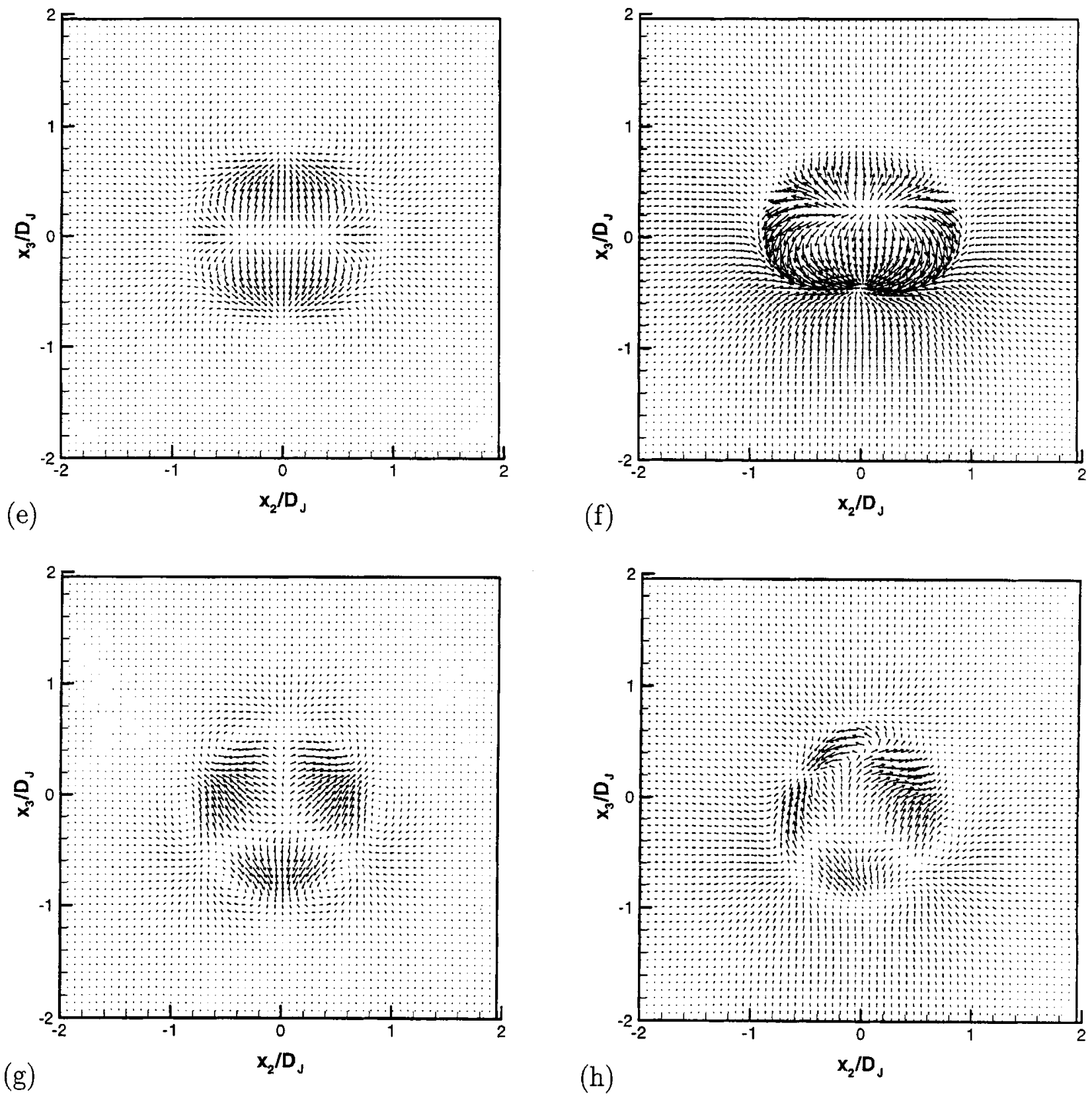

FIG. 12. (Continued.)

ing them optimal for power producing applications related to spray combustion.

\section{CONCLUSIONS}

The dispersion and mixing of evaporating liquid drops in three-dimensional circular and noncircular gaseous jets have been investigated through direct numerical simulation at Reynolds numbers characteristic to pre-transitional flows. The conservation equations were formulated in an Eulerian frame for the gas and in a Lagrangian frame for the drops, with two-way coupling whereby the flow influences the drops and the drops impact the flow through mass, momentum, species, and energy transfer. In all simulations, drops of $50 \mu \mathrm{m}$ diameter were randomly introduced at the inlet with finite relative velocity with respect to the carrier flow; the initial Stokes number was $\simeq 3$ for the value of the initial Reynolds number. The mass flow rate of drops was the same in all simulations, as were the initial Reynolds number and the Mach number of the flow. The drop initial temperature was $325 \mathrm{~K}$ and the gas temperature was $350 \mathrm{~K}$.

To quantify the influence of the drops on the flow, a separate set of simulations was performed for gaseous jets with the same geometric configuration and initial conditions. Similar to gaseous jets, two-phase flow jets with phase change were shown to reach a steady-state entrainment limit. All comparisons were performed at the time corresponding to this steady-state limit. The results show that drop-laden gaseous jets exhibit larger entrainment than their gaseous counterpart, and that this enhancement is primarily due to the 
(a)

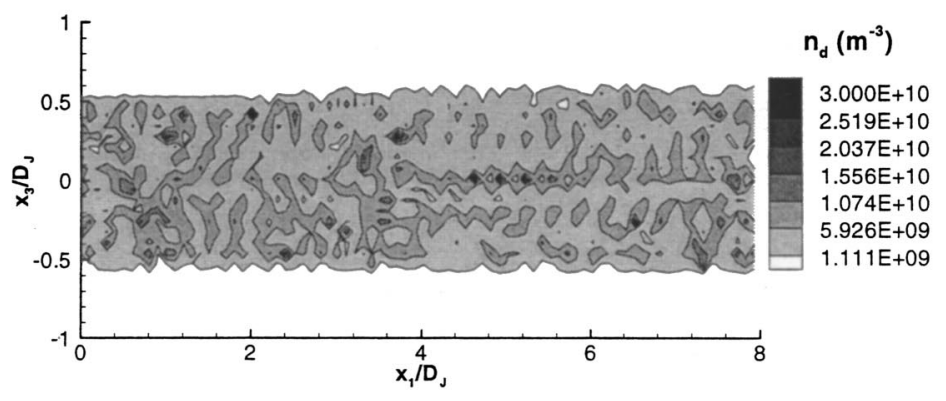

(b)

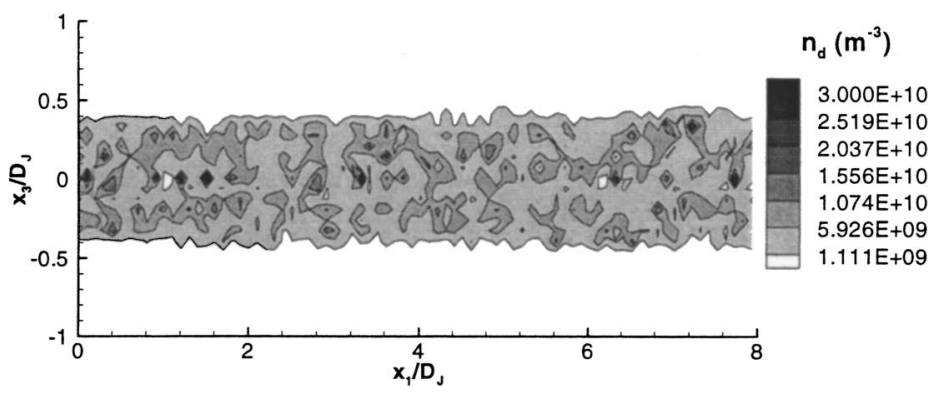

(c)

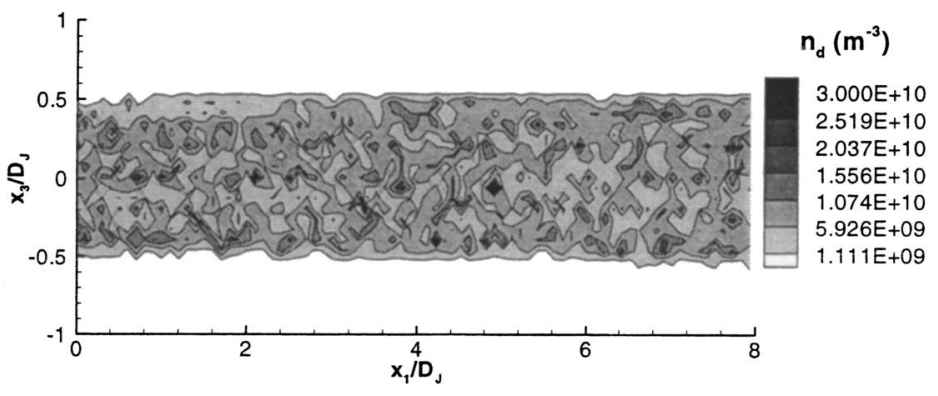

(d)

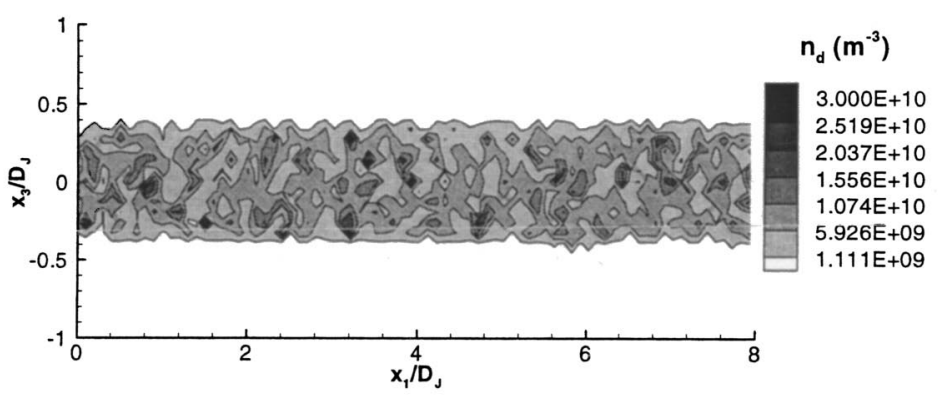

(e)

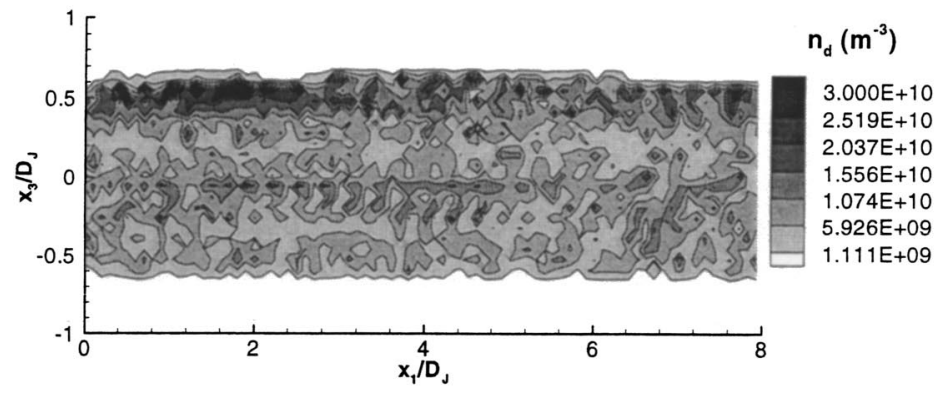

FIG. 13. Contours of instantaneous Eulerian drop-number density, $n_{d}=\Sigma_{\alpha}\left(w_{\alpha} / \Delta V_{\alpha}\right)$ at $x_{2} / D_{J}=0$ for the (a) circular jet, (b) elliptic jet, (c) square jet, (d) rectangular jet, (e) triangular jet. All at steady-state entrainment.

momentum interaction between drops and gas; for the present conditions, it is the drag rather than the evaporation term that contributes most to the momentum interaction. It is foreseen that the momentum interaction term will become even more important with increasing mass loading (the present simulations were all conducted with a fixed, modest, mass loading of 0.29). Moreover, comparisons between the potential core of gaseous and drop-laden jets revealed a re- 

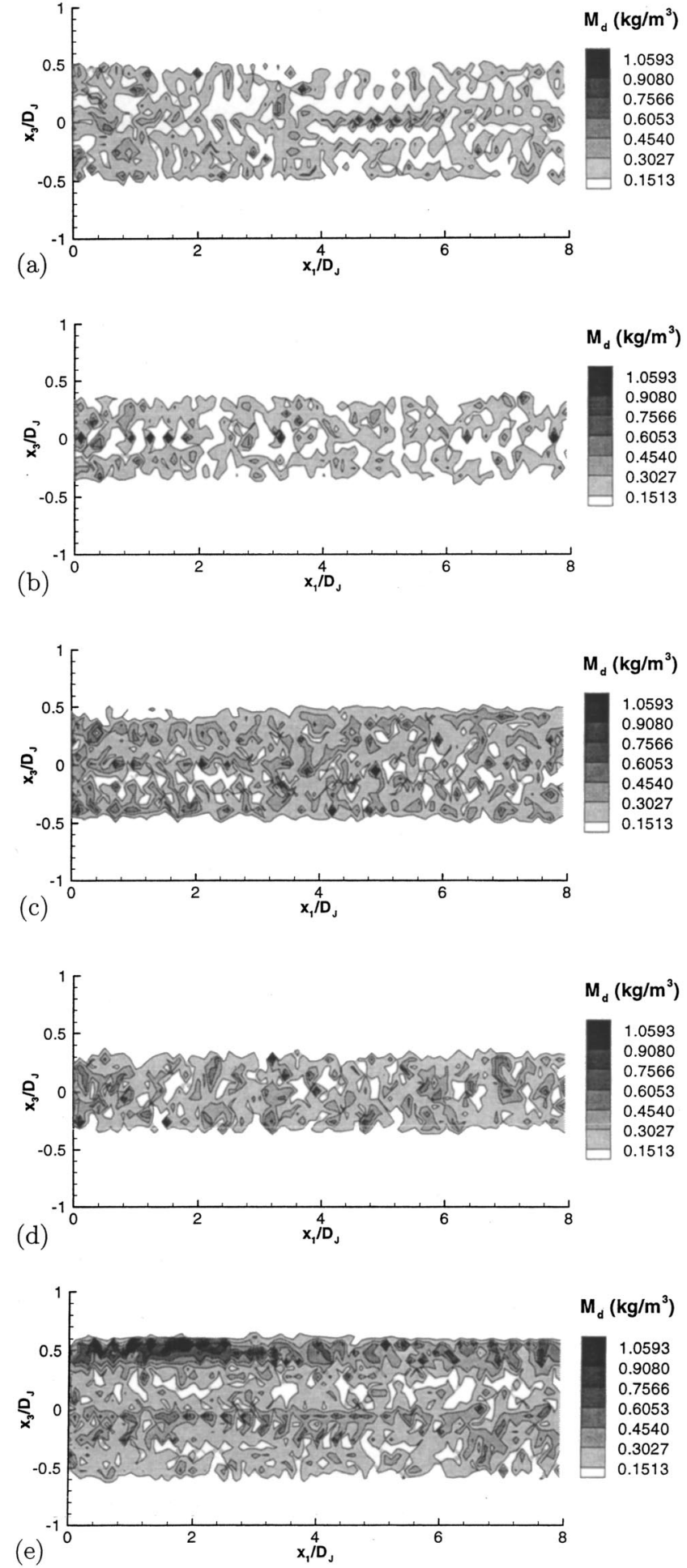

FIG. 14. Contours of instantaneous liquid mass per unit volume, $(\pi / 6) n_{d} d^{3} \rho_{L}$ at $x_{2} / D_{J}=0$ for the (a) circular jet, (b) elliptic jet, (c) square jet, (d) rectangular jet, (e) triangular jet. All at steady-state entrainment.

duction of one order of magnitude for the latter. The cores of drop-laden jets were convoluted and of similar extent for all geometries, in contrast to the regular-shape cores of gaseous jets whose extent was geometry dependent. Furthermore, none of the drop-laden jets exhibited the axis switching feature characteristic of the noncircular single-phase jets. These (a)

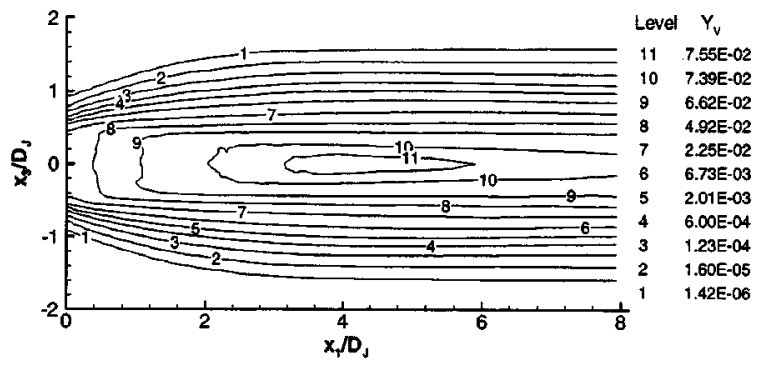

(b)

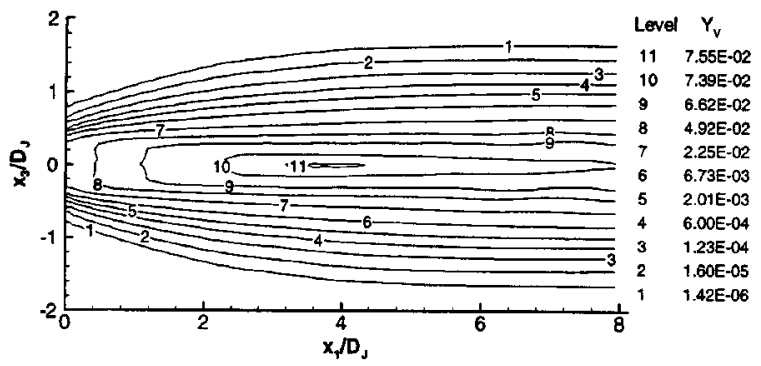

5

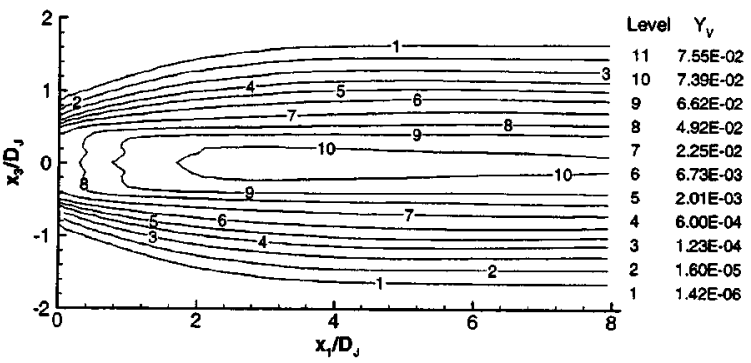

(d)

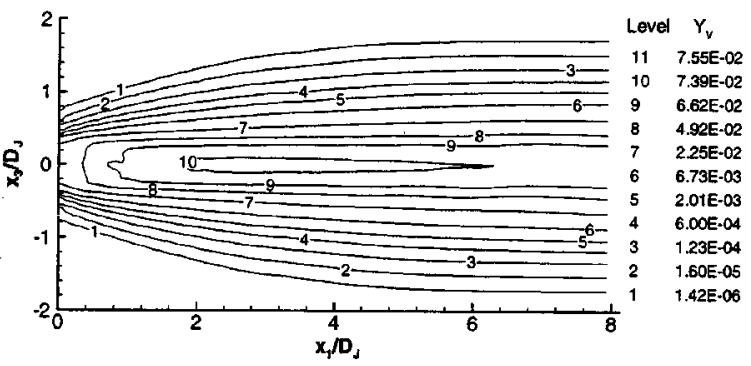

(e)

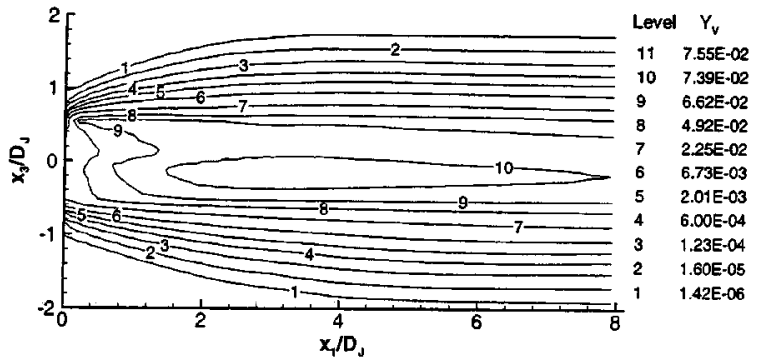

FIG. 15. Contours of the vapor mass fraction at $x_{2} / D_{J}=0$ for the (a) circular jet, (b) elliptic jet, (c) square jet, (d) rectangular jet, (e) triangular jet. All at steady-state entrainment.

aspects were attributed to the increased streamwise and spanwise vorticity in drop-laden jets.

Comparing the entrainment of drop-laden jets, the circular configuration exhibited the least entrainment, followed by the square one, while the elliptical, rectangular, and triangular configuration entrained similarly. Although the triangular jet displayed the largest fine-scale production, this occurred 
at the vertices and resulted in the accumulation of drops at those locations. Considerations of drop-number density, liquid mass, and evaporated species distributions lead to recommending the elliptic jet as the optimal configuration for combining good drop and liquid-mass dispersion with good mixing characteristics.

All these results were obtained for pre-transitional jets, and further investigations should elucidate the effect of turbulence on these findings.

\section{ACKNOWLEDGMENTS}

This investigation was performed at the Jet Propulsion Laboratory (JPL), California Institute of Technology (Caltech), and sponsored by the Department of Energy, the Hydrogen Program, under an interagency agreement with the National Aeronautics and Space Administration, with Neil Rossmeissl and Douglas Hooker as contract monitors. One of the authors (H.A-H.) would like to acknowledge financial support from the Egyptian Ministry of Education, through a graduate student scholarship during his stay at Caltech as a Special Graduate Student, covering the period of this investigation. The authors would like to thank Dr. Kenneth Harstad and Dr. Nora Okong'o of JPL for many helpful discussions on boundary condition treatments and coding aspects, respectively. The JPL supercomputer facility was used in the course of this study.

${ }^{1}$ J. J. Sangiovanni and D. S. Liscinsky, "Soot formation characteristics in well-defined spray flames," Proc. Combust. Inst. 20, 1063 (1985).

${ }^{2}$ E. Gutmark, K. C. Schadow, T. P. Parr, C. K. Harris, and K. J. Wilson, "The mean and turbulent structure of noncircular jets," AIAA-85-0543, AIAA Shear Flow Conference, March 12-14, Colorado Springs, CO (1985).

${ }^{3}$ K. C. Schadow, K. J. Wilson, D. M. Parr, C. J. Bicker, and E. Gutmark, "Reduction of flow coherence in forced subsonic jets," AIAA-85-1109, AIAA/SAE/ASME/ASEE 21st Joint Propulsion Conference and Exhibit, July 8-10, Monterrey, CA (1985).

${ }^{4}$ E. Gutmark and C-M. Ho, "Visualization of a forced elliptic jet," AIAA J. 24, 684 (1986).

${ }^{5} \mathrm{C}-\mathrm{M}$. Ho and E. Gutmark, "Vortex induction and mass entrainment in a small-aspect-ratio elliptic jet," J. Fluid Mech. 179, 383 (1987).

${ }^{6}$ E. Gutmark, K. C. Schadow, T. P. Parr, D. M. Hanson-Parr, and K. J. Wilson, "Noncircular jets in combustion systems," Exp. Fluids 7, 248 (1989).

${ }^{7}$ F. Hussain and H. S. Husain, "Elliptic jets. Part 1. Characteristics of unexcited and excited jets," J. Fluid Mech. 208, 257 (1989).

${ }^{8}$ H. S. Husain and F. Hussain, "Elliptic jets. Part 2. Dynamics of coherent structures: pairing," J. Fluid Mech. 233, 439 (1991).

${ }^{9}$ S. R. Gollahalli, T. Khanna, and N. Prahbu, "Diffusion flames of gas jets issued from circular and elliptic nozzles," Combust. Sci. Technol. 86, 267 (1992).

${ }^{10}$ F. F. Grinstein, E. Gutmark, and T. Parr, "Near field dynamics of subsonic free square jets. A computational and experimental study," Phys. Fluids 7, 1483 (1995).

${ }^{11}$ K. B. M. Q. Zaman, “Axis switching and spreading of an asymmetric jet:
The role of coherent structure dynamics," J. Fluid Mech. 316, 1 (1996).

${ }^{12}$ K. B. M. Q. Zaman, "Spreading characteristics of compressible jets from nozzles of various geometries," J. Fluid Mech. 383, 197 (1999).

${ }^{13}$ S. Koshigoe, E. Gutmark, K. C. Schadow, and A. Tubis, "Wave structures in jets of arbitrary shape. III. Triangular jet," Phys. Fluids 31, 1410 (1988).

${ }^{14}$ F. F. Grinstein and C. R. DeVore, "Coherent structure dynamics in spatially-developing square jets," AIAA-92-3441, AIAA/SAE/ASME/ ASEE 28th Joint Propulsion Conference and Exhibit, July 6-8, Nashville, TN (1992)

${ }^{15}$ F. F. Grinstein and K. Kailasanath, "Three-dimensional numerical simulations of unsteady reactive square jets," Combust. Flame 100, 2 (1995).

${ }^{16} \mathrm{C}$. K. W. Tam and A. T. Thies, "Instability of rectangular jets," J. Fluid Mech. 248, 425 (1993).

${ }^{17}$ F. F. Grinstein, "Vortex dynamics and entrainment in free rectangular jets," J. Fluid Mech. 437, 69 (2001).

${ }^{18}$ R. S. Miller, C. K. Madnia, and P. Givi, "Numerical simulation of noncircular jets," Comput. Fluids 24, 1 (1995)

${ }^{19}$ H. S. Husain and F. Hussain, "Elliptic jets. Part 3. Dynamics of preferred mode coherent structure," J. Fluid Mech. 248, 315 (1993).

${ }^{20}$ B. Rembold, N. A. Adams, and L. Kleisser, "Direct numerical simulation of a transitional rectangular jet," Int. J. Heat Fluid Flow (in press).

${ }^{21} \mathrm{X}$. Jiang and K. H. Luo, "Spatial DNS of flow transition of a rectangular buoyant reacting free-jet," Journal of Turbulence 2, 1 (2001).

${ }^{22}$ E. Gutmark and F. F. Grinstein, "Flow control with noncircular jets," Annu. Rev. Fluid Mech. 31, 239 (1999).

${ }^{23}$ R. S. Miller and J. Bellan, "Direct numerical simulation of a confined three-dimensional gas mixing layer with one evaporating hydrocarbondroplet laden stream," J. Fluid Mech. 384, 293 (1999).

${ }^{24}$ R. S. Miller and J. Bellan, "Direct numerical simulation and subgrid analysis of a transitional droplet laden mixing layer," Phys. Fluids 12, 650 (2000).

${ }^{25} \mathrm{~N}$. Okong'o and J. Bellan, "A priori subgrid analysis of temporal mixing layers with evaporating droplets," Phys. Fluids 12, 1573 (2000).

${ }^{26}$ K. D. Squires and J. K. Eaton, "Particle response and turbulence modification in isotropic turbulence," Phys. Fluids A 2, 1191 (1990).

${ }^{27}$ K. D. Squires and J. K. Eaton, "Preferential concentration of particles by turbulence," Phys. Fluids A 3, 1169 (1991).

${ }^{28}$ L. M. Liljegren, "On modeling particles as point forces in gas-particle flows," ASME FED 236, 173 (1996).

${ }^{29}$ R. S. Miller, K. Harstad, and J. Bellan, "Evaluation of equilibrium and non-equilibrium evaporation models for many droplet gas-liquid flow simulations," Int. J. Multiphase Flow 24, 1025 (1998).

${ }^{30}$ W. E. Ranz and W. R. Marshall, "Evaporation from drops: I," Chem. Eng. Prog. 48, 141 (1952).

${ }^{31}$ W. E. Ranz and W. R. Marshall, "Evaporation from drops: II," Chem. Eng. Prog. 48, 173 (1952).

${ }^{32}$ J. O. Hirshfelder, C. F. Curtis, and R. B. Bird, Molecular Theory of Gases and Liquids (Wiley, New York, 1954), pp. 516, 717.

${ }^{33}$ T. J. Poinsot and S. K. Lele, "Boundary conditions for direct simulations of compressible viscous flows," J. Comput. Phys. 101, 104 (1992).

${ }^{34} \mathrm{M}$. Baum, T. Poinsot, and D. Thévenin, "Accurate boundary conditions for multicomponent reactive flows," J. Comput. Phys. 116, 247 (1994).

${ }^{35} \mathrm{C}$. Kennedy and M. Carpenter, "Several new numerical methods for compressible shear layer simulations," Appl. Numer. Math. 14, 397 (1994).

${ }^{36} \mathrm{X}-\mathrm{L}$. Tong and L-P. Wang, "Direct simulations of particle transport in two and three dimensional mixing layers," ASME Fluids Engineering Division Summer Meeting, paper 3635, June 22-26 (1997).

${ }^{37} \mathrm{D}$. Liepmann and M. Gharib, "The role of streamwise vorticity in the near-field entrainment of round jets," J. Fluid Mech. 245, 643 (1992).

${ }^{38}$ V. G. McDonell and G. S. Samuelsen, "Structure of vaporizing pressure atomized sprays," Atomization Sprays 3, 321 (1993). 